\title{
ADHFE1 is a breast cancer oncogene and induces metabolic reprogramming
}

\author{
Prachi Mishra, ${ }^{1}$ Wei Tang, ${ }^{1}$ Vasanta Putluri, ${ }^{2,3}$ Tiffany H. Dorsey, ${ }^{1}$ Feng Jin, ${ }^{2,3}$ Fang Wang, ${ }^{4}$ Donewei Zhu, ${ }^{4}$ Lauren Amable, ${ }^{5}$ \\ Tao Deng, ${ }^{6}$ Shaofei Zhang, ${ }^{6}$ J. Keith Killian, ${ }^{7}$ Yonghong Wang, ${ }^{7}$ Tsion Z. Minas, ${ }^{1}$ Harry C. Yfantis, ${ }^{8}$ Dong H. Lee, ${ }^{8}$ \\ Arun Sreekumar, ${ }^{2}$ Michael Bustin, ${ }^{6}$ Wei Liu, ${ }^{4}$ Nagireddy Putluri, ${ }^{2,3}$ and Stefan Ambs ${ }^{1}$ \\ 'Laboratory of Human Carcinogenesis, Center for Cancer Research (CCR), National Cancer Institute (NCI), NIH, Bethesda, Maryland, USA. ²Department of Molecular and Cell Biology, Verna and Marrs McLean \\ Department of Biochemistry and Alkek Center for Molecular Discovery, and ${ }^{3}$ Advanced Technology Core, Baylor College of Medicine, Houston, Texas, USA. ${ }^{4}$ Agios Pharmaceuticals, Cambridge, Massachusetts, \\ USA. ${ }^{5}$ National Institute of Minority Health and Health Disparities, ${ }^{6}$ Protein Section, Laboratory of Metabolism, CCR, NCl, and ${ }^{7}$ Cenetics Branch, CCR, and Clinical Molecular Profiling Core, NCI, NIH, Bethesda, \\ Maryland, USA. ${ }^{8}$ Pathology and Laboratory Medicine, Baltimore Veterans Affairs Medical Center, Baltimore, Maryland, USA.
}

\begin{abstract}
Metabolic reprogramming in breast tumors is linked to increases in putative oncogenic metabolites that may contribute to malignant transformation. We previously showed that accumulation of the oncometabolite, 2-hydroxyglutarate (2HG), in breast tumors was associated with MYC signaling, but not with isocitrate dehydrogenase (IDH) mutations, suggesting a distinct mechanism for increased 2HG in breast cancer. Here, we determined that D-2HG is the predominant enantiomer in human breast tumors and show that the D-2HG-producing mitochondrial enzyme, alcohol dehydrogenase, iron-containing protein 1 (ADHFE1), is a breast cancer oncogene that decreases patient survival. We found that MYC upregulates ADHFE1 through changes in iron metabolism while coexpression of both ADHFE1 and MYC strongly enhanced orthotopic tumor growth in MCF7 cells. Moreover, ADHFE1 promoted metabolic reprogramming with increased formation of D-2HG and reactive oxygen, a reductive glutamine metabolism, and modifications of the epigenetic landscape, leading to cellular dedifferentiation, enhanced mesenchymal transition, and phenocopying alterations that occur with high D-2HG levels in cancer cells with IDH mutations. Together, our data support the hypothesis that ADHFE1 and MYC signaling contribute to D-2HC accumulation in breast tumors and show that D-2HG is an oncogenic metabolite and potential driver of disease progression.
\end{abstract}

\section{Introduction}

Breast tumors undergo metabolic reprogramming as part of malignant transformation $(1,2)$. This reprogramming leads to increased availability of putative oncogenic metabolites that are used for the synthesis of biomolecules, energy metabolism, or as signaling molecules $(3,4)$. Cancer metabolism has also been shown to modify the chromatin of cells, the tumor microenvironment, or antitumor immunity (5). We and others reported that metabolite patterns of breast tumors are greatly different from those in the adjacent noncancerous tissue and vary between disease subtypes (6-8). A key discovery from these studies was the observation that 2-hydroxyglutarate (2HG) can accumulate in breast tumors $(7,8)$. These tumors did not harbor the isocitrate dehydrogenase 1 and 2 (IDH1 and -2) mutations that have previously been linked to $2 \mathrm{HG}$ accumulation in gliomas and leukemia $(9,10)$, indicating that an alternative mechanism must lead to increased $2 \mathrm{HG}$ in breast cancer. We also observed that aberrant $2 \mathrm{HG}$ accumulation is mainly seen in tumors with a MYC signaling signature and found that knockdown of alcohol dehydrogenase, iron-containing protein 1 (ADHFE1) decreased 2HG in breast cancer cells (7). However, these studies neither identified the enantiomeric form of $2 \mathrm{HG}$

Conflict of interest: F. Wang, D. Zhu, and W. Liu are employees of Agios Pharmaceuticals. Submitted: March 8, 2017; Accepted: October 17, 2017.

Reference information: J Clin Invest. 2018;128(1):323-340.

https://doi.org/10.1172/JCI93815. in breast tumors, nor did they characterize ADHFE1. Here, we tested the hypothesis that D-2HG-producing ADHFE1 is a breast cancer oncogene that alters tumor metabolism. We show that $\mathrm{D}-2 \mathrm{HG}$ is the predominant $2 \mathrm{HG}$ enantiomer in breast tumors and describe ADHFE1 as a MYC-linked and iron-inducible oncogene that promotes metabolic reprogramming with an increase in D-2HG, reactive oxygen species (ROS) formation, and histone methylation, leading to cellular dedifferentiation and mesenchymal transition. We also show that D-2HG by itself induces these phenotypes, including reductive carboxylation of glutamine and an increase in mitochondrial ROS.

\section{Results}

$D-2 H G$ is the predominant enantiomer elevated in human breast tumors. We and others previously reported that total $2 \mathrm{HG}$ accumulates in a subset of human breast tumors that were primarily estrogen receptor-negative (ER-negative) $(7,8)$. Here, we interrogated the enantiomeric forms of $2 \mathrm{HG}$ in $15 \mathrm{ER}$-negative human breast tumors using enantiomer-specific targeted mass spectrometry (Figure 1A). Consistent with our earlier data, 2HG levels were elevated in these tumors. Notably, most of the tumors contained accumulated levels of the D-enantiomer while few of them contained both D- and L-2HG at similar levels (Figure 1A), indicating the existence of multiple pathways that could lead to increased $2 \mathrm{HG}$ formation in these tumors. In adjacent noncancerous tissues $(n=4), \mathrm{D}-2 \mathrm{HG}$ averaged $0.18 \pm 0.055 \mathrm{mg} / \mathrm{kg}$ compared with 1.28 
$\mathrm{mg} / \mathrm{kg}$ for the tumor with the lowest D-2HG accumulation (7.1fold increase in tumor) and with $26.3 \mathrm{mg} / \mathrm{kg}$ for the tumor with the highest D-2HG accumulation (147.8-fold increase in tumor).

Relationship between MYC and ADHFE1. 2HG accumulation in breast tumors is associated with a MYC activation signature, while knockdown of ADHFE1 decreased intracellular 2HG levels in ER-negative breast cancer cells (7). Based on these previous findings, we hypothesized that ADHFE1 is a MYC-linked candidate oncogene that promotes $\mathrm{D}-2 \mathrm{HG}$ production in mammary epithelial cells. To test the association of ADHFE1 with MYC, we queried The Cancer Genome Atlas (TCGA) breast cancer data set (at http://www.cbioportal.org/public-portal), which revealed a significant co-occurrence of ADHFE1 amplifications at 8q12.3 and MYC amplifications at $8 \mathrm{q} 24$ in a subset of breast tumors (Figure 1B). An additional analysis of the METABRIC breast cancer data set (11) showed that patients with ADHFE1 amplifications in their tumors experience a moderately decreased survival (Supplemental Figure 1; supplemental material available online with this article; https://doi.org/10.1172/JCI93815DS1). However, high ADHFE1 protein expression predicted poor survival of patients with ERnegative breast tumors (Figure 1C) in a Maryland breast cancer cohort (12). This association of ADHFE1 with breast cancer survival was independent of age, race/ethnicity, disease stage, therapy, and MYC protein expression in the multivariable survival analysis (hazard ratio [HR]: 2.61; 95\% confidence interval: 1.15 to 5.95 for high vs. low ADHFE1 expression). To further explore the relationship between MYC and ADHFE1, we induced MYC signaling in human mammary epithelial cells (HMEC-MYC) using 4-hydroxytamoxifen (13) or downregulated endogenous MYC in SUM159T breast cancer cells using an inducible shRNA system. Consistent with a relationship between MYC and ADHFE1, induction of MYC signaling in HMEC-MYC increased ADHFE1, while MYC knockdown in SUM159T cells reduced ADHFE1 expression (Figure 1D). Additionally, we overexpressed human ADHFE1 in a malignant (MCF7) and 2 nonmalignant breast epithelial cell lines (MCF10A and MCF12A), all having low endogenous ADHFE1 expression, using a lentiviral expression construct for human $A D H F E 1$ (Figures $1 \mathrm{D}$ and Supplemental Figure 2). Upregulation of ADHFE1 in these cell lines also increased MYC (Figure 1D), suggesting the existence of a mutual regulatory loop between MYC and ADHFE1 in breast cancer. Together, our findings suggest a potential oncogenic role of ADHFE1 in breast cancer and disease progression.

ADHFE1 promotes orthotopic tumor growth. To examine whether ADHFE1 is an oncogene that enhances tumor growth, MCF7 cells over-expressing ADHFE1, MYC, or both ADHFE1 and MYC, were injected into the mammary fat pad of NOD/SCID gamma mice. Expression of either ADHFE1 or MYC significantly increased orthotopic tumor growth of these cells in the mammary gland, but the coexpression of $A D H F E 1$ and $M Y C$ enhanced tumor growth more than the expression of either one of the 2 genes alone (Figure $1 \mathrm{E}$ ), indicating that the coamplification of ADHFE1 and $M Y C$, which we observed in a subset of human breast tumors (Figure 1B), may lead to a growth advantage. MCF7 tumors that coexpressed ADHFE1 and MYC also had the highest levels of 2HG and 4-hydroxybutyrate, a substrate for ADHFE1 to produce $2 \mathrm{HG}$ (Figure $1 \mathrm{~F}$ ).

MYC upregulates ADHFE1 through increased iron metabolism. The amplification of the ADHFE1 locus in a subset of breast tumors and the induction ADHFE1 expression by MYC in cell lines suggest the existence of different mechanisms that may upregulate ADHFE1. Therefore, we explored both the relationship between ADHFE1 genomic amplification and ADHFE1 protein expression in human breast tumors and the effect of MYC on ADHFE1 expression. To correlate ADHFE1 genomic amplification and ADHFE1 protein expression in breast tumors, we obtained TCGA breast cancer data for mass spectrometry-based global protein expression (14), and the gene copy number status in the tumors, from cbioportal (http://www.cbioportal.org/). In a combined analysis of all breast tumors, we did not find that a genomic amplification of ADHFE1 significantly correlates with ADHFE1 protein expression. Yet, in the analysis of the basal-like subset of breast tumors $(n=24)$, which is the subtype with the most significant increase in 2HG (7), ADHFE1 genomic amplification positively correlated with ADHFE1 $(\rho=0.38, P<0.05)$ and vimentin $(\rho=0.48, P<$ $0.05)$ protein expression and inversely correlated with E-cadherin expression $(\rho=-0.32, P<0.05)$, indicating that an amplification may increase protein expression and epithelial-to-mesenchymal transition (EMT) in this subtype. However, the moderate strength of the correlation between ADHFE1 amplification and protein expression shows that additional mechanisms of ADHFE1 upregulation must exist in breast tumors. Thus, we examined whether MYC induces ADHFE1 expression. We did not find that MYC directly regulates $A D H F E 1$ promoter activity using a reporter assay (Figure 2A), although a MYC binding site exists upstream of the human ADHFE1 promoter region (Supplemental Figure 3). Instead, further investigations discovered a mechanism by which MYC upregulates ADHFE1 through an increase in iron metabolism. MYC regulates expression of iron metabolism-related genes (15). We found that MYC induces these genes in HMEC-MYC cells and increases iron accumulation in mitochondria, where ADHFE1 is located, and also show that $\mathrm{Fe}^{2+}$ induces ADHFE1 expression (Figures 2, B-D), consistent with a previous report (16). Furthermore, we show that a chelator of $\mathrm{Fe}^{2+}, 3$-aminopyridine-2-carboxaldehyde thiosemicarbazone (3-AP), inhibits $\mathrm{FeSO}_{4}$-induced and MYC-induced upregulation of ADHFE1 (Supplemental Figure 4 and Figure 2E). In summary, these data indicate that increased MYC signaling in breast tumors can upregulate ADHFE1 through its effects on iron metabolism, and that ADHFE1 amplifications may also contribute to increased ADHFE1 protein expression in a subset of breast tumors.

Metabolic reprogramming associated with ADHFE1 and D-2HG in breast cancer. ADHFE1 is a mitochondrial enzyme (EC 1.1.99.24) that catalyzes the oxidation of 4-hydroxybutyrate to succinic semialdehyde coupled to the reduction of $\alpha$-ketoglutarate to D-2HG $(17,18)$, but may also use other substrates, such as 3-hydroxypropionate (19). Consistent with this catalytic reaction, we found that levels of 4-hydroxybutyrate and 2HG correlated in the MCF7 xenograft tumors (Figure 1F) and in human breast tumors. Analyzing our publicly available metabolome data for breast cancer (7), we noticed that 4-hydroxybutyrate is markedly increased in ER-negative breast tumors (7.1-fold when compared with adjacent noncancerous tissue; $P<0.01)$ and significantly correlated with 2HG levels in these tumors (Spearman's $\rho=0.5$; both $P<0.01)$. One reason why 4 -hydroxybutyrate and 2 HG simultaneously increase in breast tumors could be MYC signaling. As shown 


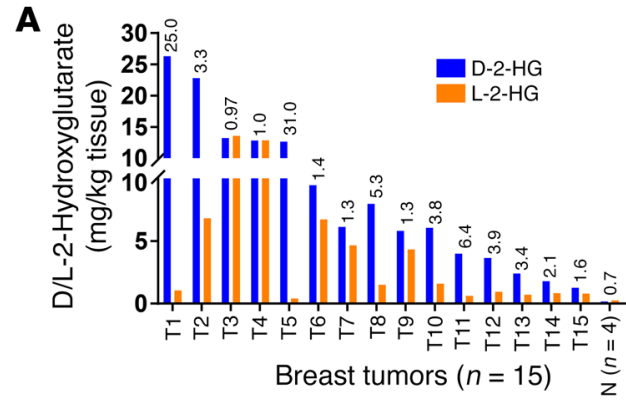

B Cooccurence of MYC and ADHFE1 amplification: $\log$ odds ratio $>3, P<0.01$

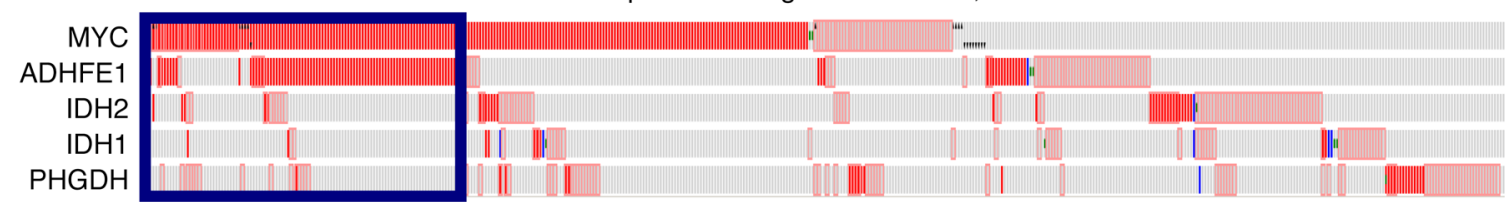

C

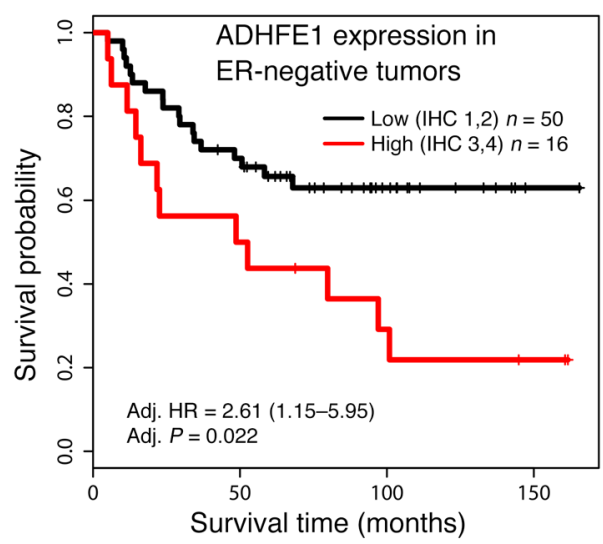

D

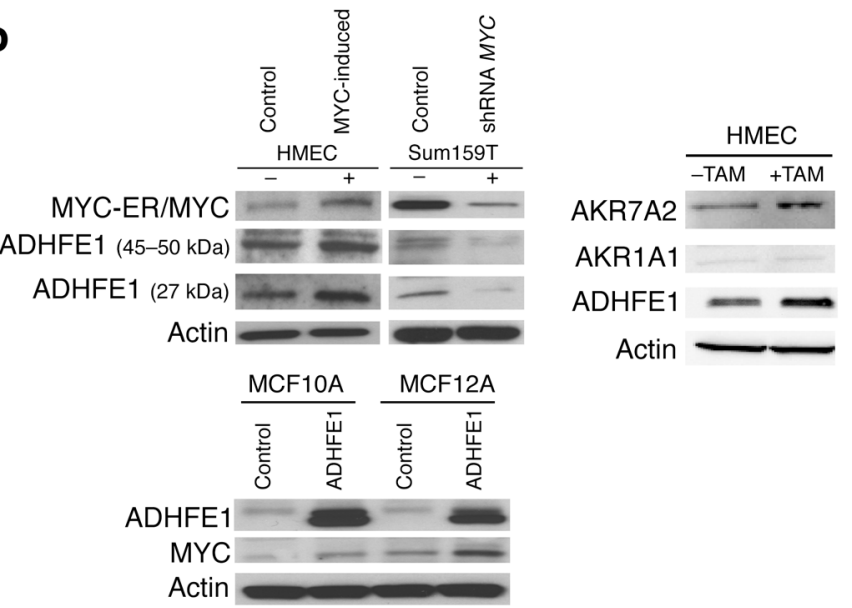

E

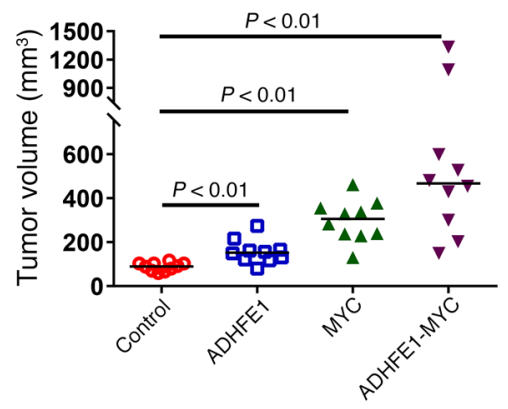

$\mathbf{F}$

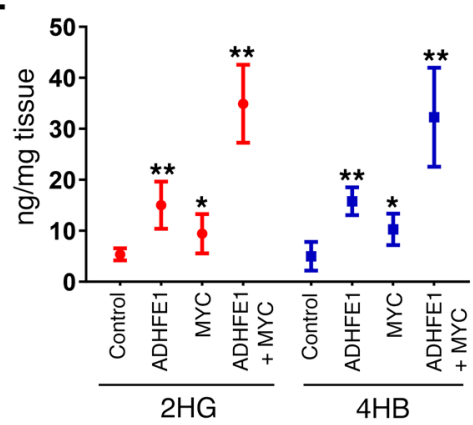

Figure 1. Co-occurrence of ADHFE1 and MYC amplifications in human breast tumors and accelerated tumor growth of ADHFE1-overexpressing MCF7 cells. (A) Accumulation of $D$ - and $L$-2-hydroxyglutarate and their ratio ( $D$ to $L$ ratio above bars) in 15 human breast tumors and 4 adjacent noncancerous breast tissues (N). (B) Co-occurrence of ADHFE1 and MYC amplifications in human breast tumors $(P<0.001)$. Shown are 473 TCGA breast tumors with amplification (dark red) or overexpression (light red) of 1 of the 5 listed genes previously associated with D-2HG. Blue bars: deletion or reduced expression. IDH, isocitrate dehydrogenase; PHGDH, phosphoglycerate dehydrogenase. (C) High ADHFE1 protein expression by immunohistochemistry (IHC) is associated with decreased survival of patients with ER-negative breast cancer. $P=0.012$ by the log-rank test. HR, hazard ratio. (D) Western blots. Top left panel: MYC upregulates ADHFE1. MYC signaling was induced (MYC-ER fusion in HMEC-MYC) or suppressed (inducible shRNA in SUM159T) with 4-hydroxytamoxifen (+) or doxycycline (+), respectively. HMEC, human mammary epithelial cell. Lower panel: ADHFE1 transgene expression in MCF10A and MCF12A cells increases MYC. Right panel: Induction of aldo-keto reductase AKR7A2, but not AKR1A1, in HMEC-MYC cells after 4-hydroxytamoxifen-stimulated MYC signaling (+TAM). (E) Increased tumor growth of MCF7 cells over-expressing either ADHFE1, MYC, or both ADHFE1 and MYC (ADHFE1-MYC). Solid lines show median for each group. Tumor growth was measured 16 weeks after injection of MCF7 cells into mammary fat pads. Two-sided $t$ test for comparisons with control group ( $n=10$ per group). $P=0.05$ for MYC versus ADHFE1-MYC tumors. (F) Increased 2-hydroxyglutarate (2HG) and 4-hydroxybutyrate (4HB) levels in MCF7 tumors overexpressing ADHFE1 and MYC ( $n=10$ per group). ${ }^{*} P<0.05,{ }^{* *} P<0.01$, versus control group using 2 -sided $t$ test. Shown is the mean \pm SD. ANOVA test for differences between groups was used in $\mathbf{E}$ and $\mathbf{F}: P<0.001$. 

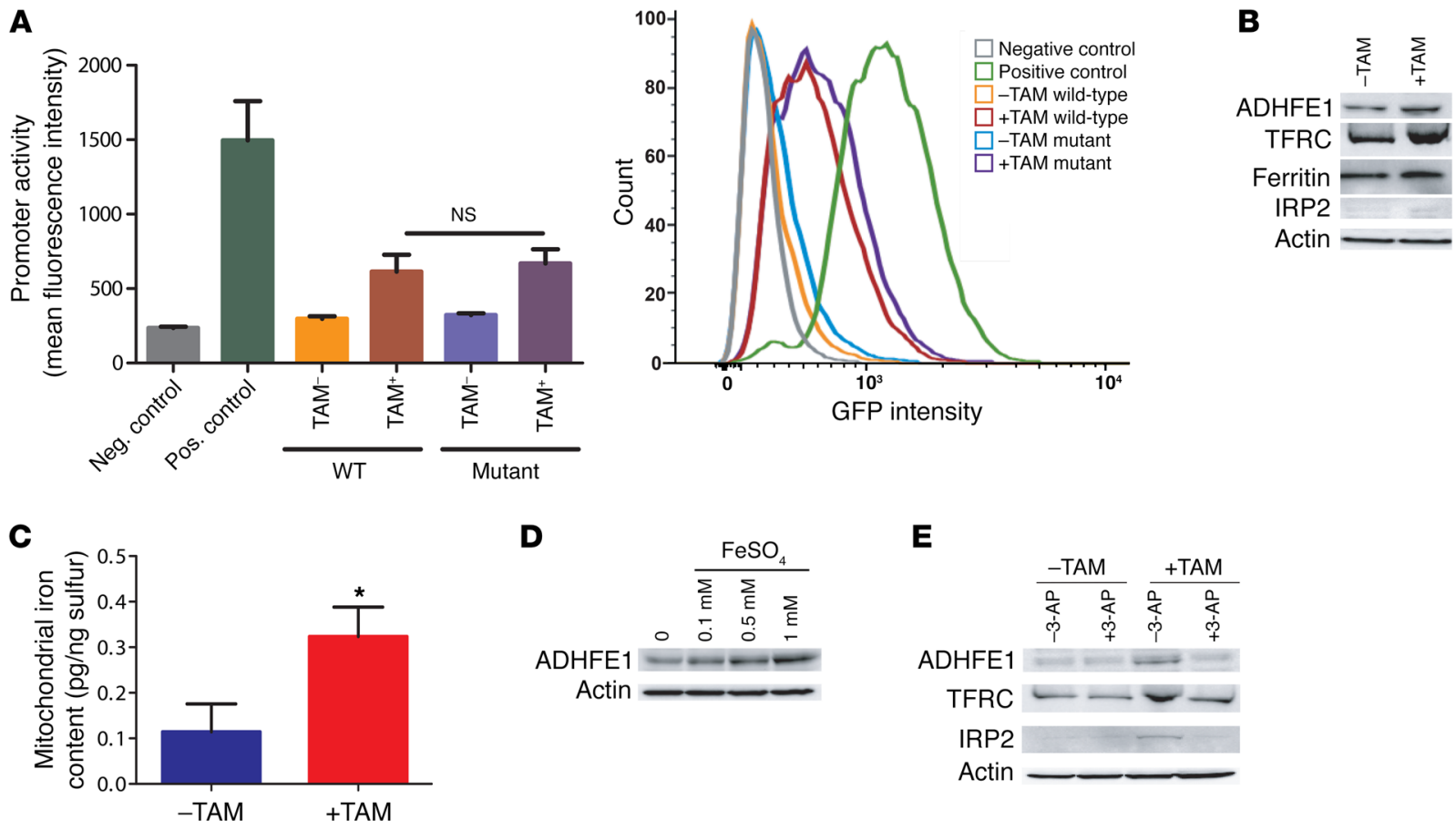

D

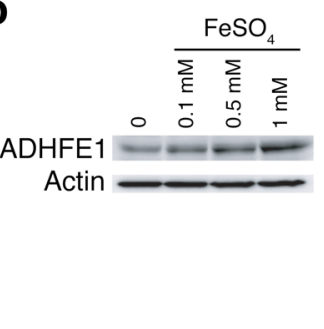

$\mathbf{E}$

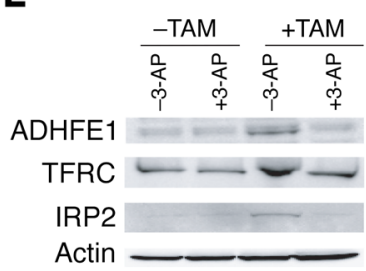

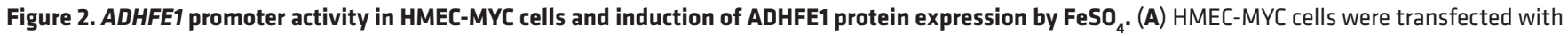
a reporter construct containing a 5-kb segment of the human ADHFE1 promoter region with a MYC binding site. Cells were transfected with constructs containing either the intact MYC binding site (WT) or a mutated sequence (mutant), as described in Methods. MYC signaling was induced with 4-hydroxytamoxifen (+TAM). Addition of TAM modestly increased ADHFE1 promoter activity but the activity was not dependent on the presence of an intact MYC binding site. Shown is the mean \pm SD for triplicate experiments. Two-sided $t$ test; NS, not significantly different. (B) MYC signaling (+TAM) increases expression of iron metabolism genes in HMEC-MYC cells. (C) MYC signaling also increases mitochondrial iron content in these cells. $n=4$. Shown is the mean \pm SD. ${ }^{*} P<0.05$ (2-sided $t$ test). (D) Treatment of HMECs with $\mathrm{FeSO}_{4}$ for 24 hours increases ADHFE1 expression. (E) Induction of ADHFE1 and iron metabolism genes by MYC signaling in HMEC-MYC cells can be inhibited by the Fe ${ }^{2+}$ chelator, 3-AP $(0.5 \mu \mathrm{M})$, which was added together with TAM. In B, C, and E, TAM treatment was for 96 hours. TFRC, transferrin receptor protein 1; IRP2, iron-responsive element-binding protein 2.

in Figure 1D, MYC induced not only ADHFE1 in HMEC-MYC cells but also the aldo-keto reductase AKR7A2, a key enzyme in the synthesis of 4-hydroxybutyrate in the brain (20). Additionally, ENCODE ChIP-seq data show that MYC has several binding sites in the AKR7A2 promoter region (Supplemental Figure 5).

Besides the formation of D-2HG, ADHFE1 may affect breast cancer metabolism more broadly. Therefore, we performed a metabolome analysis of cancer-related metabolites in ADHFE1overexpressing cells, as described under Methods. In the 3 cell lines, ADHFE1 increased both intracellular and secreted D-2HG under aerobic and hypoxic conditions (Supplemental Figure 6). In all of them, D-2HG accumulation was higher under hypoxic than aerobic conditions and reached an upper limit at 10- to 15 -fold relative to the control cells. Further examination of 116 metabolites in MCF12A cells revealed a very distinct pattern of metabolic changes in ADHFE1-overexpressing cells. Marker metabolites for glycolysis, TCA cycle, and polyamine synthesis were elevated in these cells and acetyl-CoA increased 14.3-fold over control levels (Figure 3 and Supplemental Figure 7). Moreover, the NADPH/NADP ration was elevated (Supplemental Figure 7), and TCA metabolites that would be generated by a reductive glutamine metabolism accumulated, whereas other TCA cycle-related metabolites remained unchanged (Figure 3B). We confirmed this metabolic rewiring in mitochondria associated with $A D H F E 1$ overexpression using a ${ }^{13} \mathrm{C}$-labeled glutamine isotopomer method. Our studies revealed an increased incorporation of the $1{ }^{13} \mathrm{C}$ label into citrate and malate in MCF10A and MCF12A cells with upregulated ADHFE1 (Figure 4, A-E). These findings support a role of ADHFE1 in promoting a reductive glutamine metabolism (Figure 4F), also termed reductive carboxylation (21), in which glutamine-derived $\alpha$-ketoglutarate is metabolized into isocitrate and citrate for increased acetyl-CoA synthesis and lipogenesis. Because intracellular D-2HG in itself may promote a reductive glutamine metabolism, we added cell-permeant D-2HG to MCF10A and MCF12A cells and cultured the cells in presence of $\left[1-{ }^{13} \mathrm{C}\right]$ glutamine. This experiment revealed an increased incorporation of the $1{ }^{13} \mathrm{C}$ label into citrate (Supplemental Figure 8), consistent with an upregulation of reductive carboxylation by D-2HG. Thus, the increase in D-2HG in ADHFE1-overexpressing cells may contribute to the changes in metabolism due to this enzyme. Lastly, we assessed the incorporation of glutamine labeled at all 5 carbons with ${ }^{13} \mathrm{C}\left(\left[1-5{ }^{13} \mathrm{C}\right]\right.$ glutamine) into succinate and citrate in MCF10A cells treated with cell-permeant D-2HG (1 mM octyl-D-2HG for 48 hours). This experiment showed that D-2HG significantly decreases the flux of $\left[1-5{ }^{13} \mathrm{C}\right]$ glutamine into succinate while increasing the flux into citrate via reductive carboxylation as shown by the increased fraction of $\left[1-5{ }^{13} \mathrm{C}\right]$-labeled citrate in these cells (Figure $4 \mathrm{G}$ ), indicating that D-2HG may inhibit the $\alpha$-ketoglutarate dehydrogenase complex and thereby increase reductive carboxylation. 
A

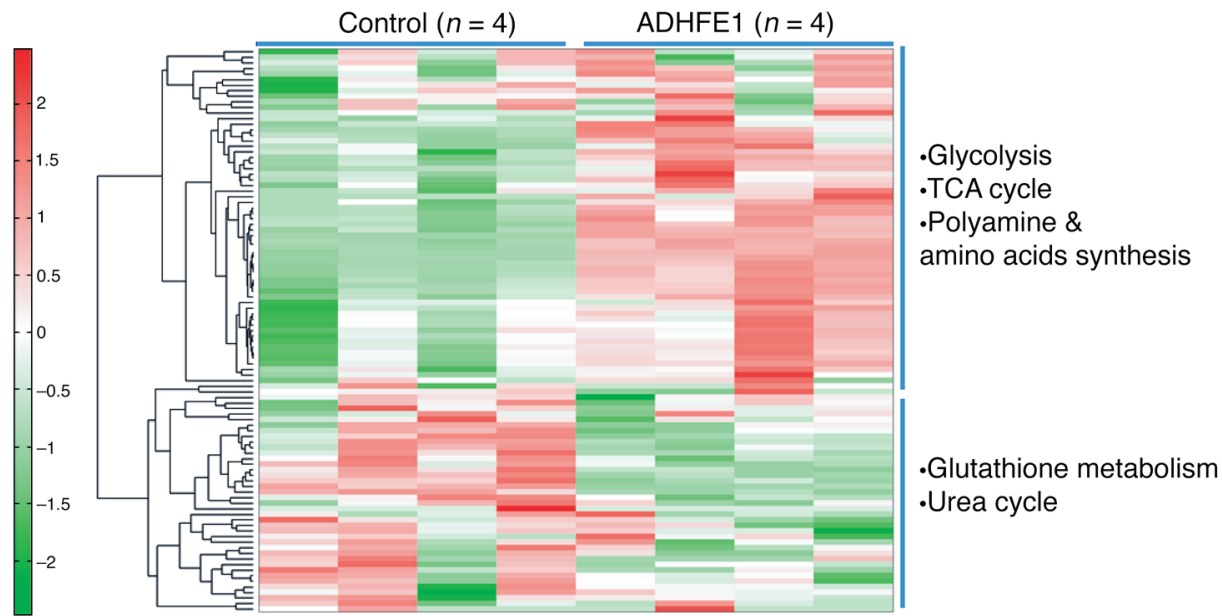

B
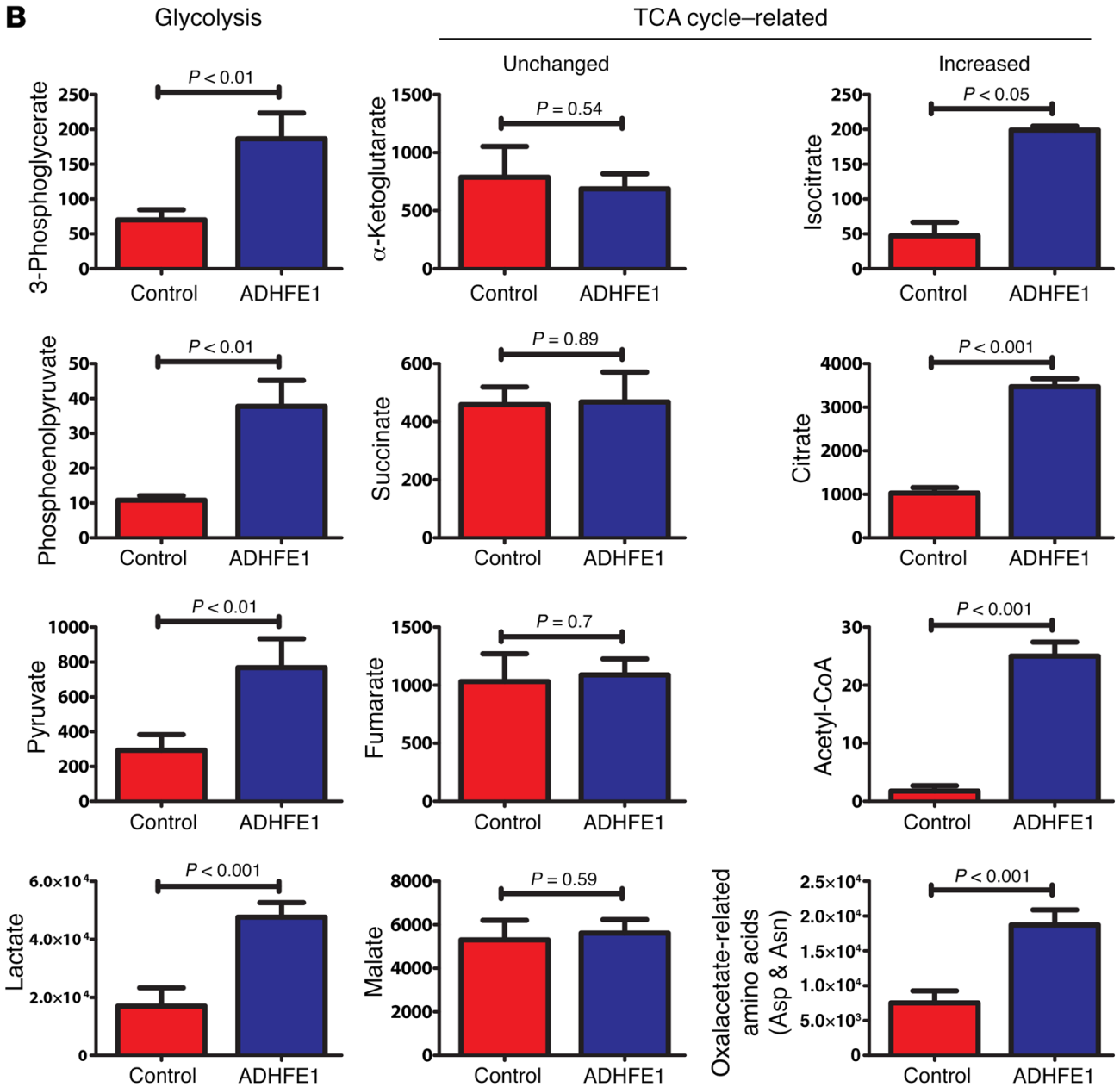

Figure 3. Metabolic reprogramming in MCF12A cells with upregulated ADHFE1. (A) Heatmap showing increased abundance of glycolysis, tricarboxylic acid (TCA) cycle, polyamines, and amino acid-related metabolites (red) and decreased abundance of glutathione and urea cycle-related metabolites (green) in ADHFE1-expressing MCF12A cells.

(B) Increased abundance of glycolytic metabolites and TCA cyclerelated metabolites that can be generated by a reductive glutamine metabolism ("increased") in cells with upregulated ADHFE1. Absolute quantification: metabolite concentrations are reported as $\mathrm{pmol} / 10^{6}$ cells (mean $\pm \mathrm{SD}, n=4$ ). Two-sided $t$ test was used to determine statistical significance.
Since a retrograde TCA cycle provides cells with a survival advantage under hypoxia $(21,22)$, we examined if ADHFE1-overexpressing cells have a growth advantage over vector control cells when cultured under hypoxic conditions. As shown in Supplemental Figure 9, ADHFE1 led to a growth advantage of MCF10A and MCF12A cells only in the hypoxic state, but not in the normoxic state, when compared with controls.

$A D H F E 1$ and D-2HG induce EMT. Not much is known about the function of ADHFE1 beyond its role as a mitochondrial enzyme and its increased methylation in colorectal cancer (23). To gain a deeper understanding of ADHFE1's role in cancer, we generated gene expression profiles from vector control and ADHFE1-overexpressing MCF7 and MCF12A cells (Supplemental Tables 1 and 2) and examined the respective gene expression signatures for pathway associations. In parallel, we generated expression profiles from cells treated with either $1 \mathrm{mM}$ cell-permeant D-2HG (octyl-2HG) or the control compound, pentanedioic acid monooctylester (PAMO) (Supplemental Tables 3 and 4), which has permeability charac- 
A

MCF10A

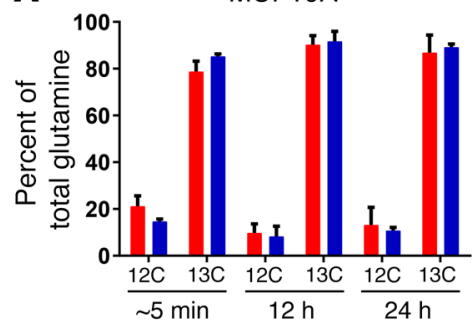

B
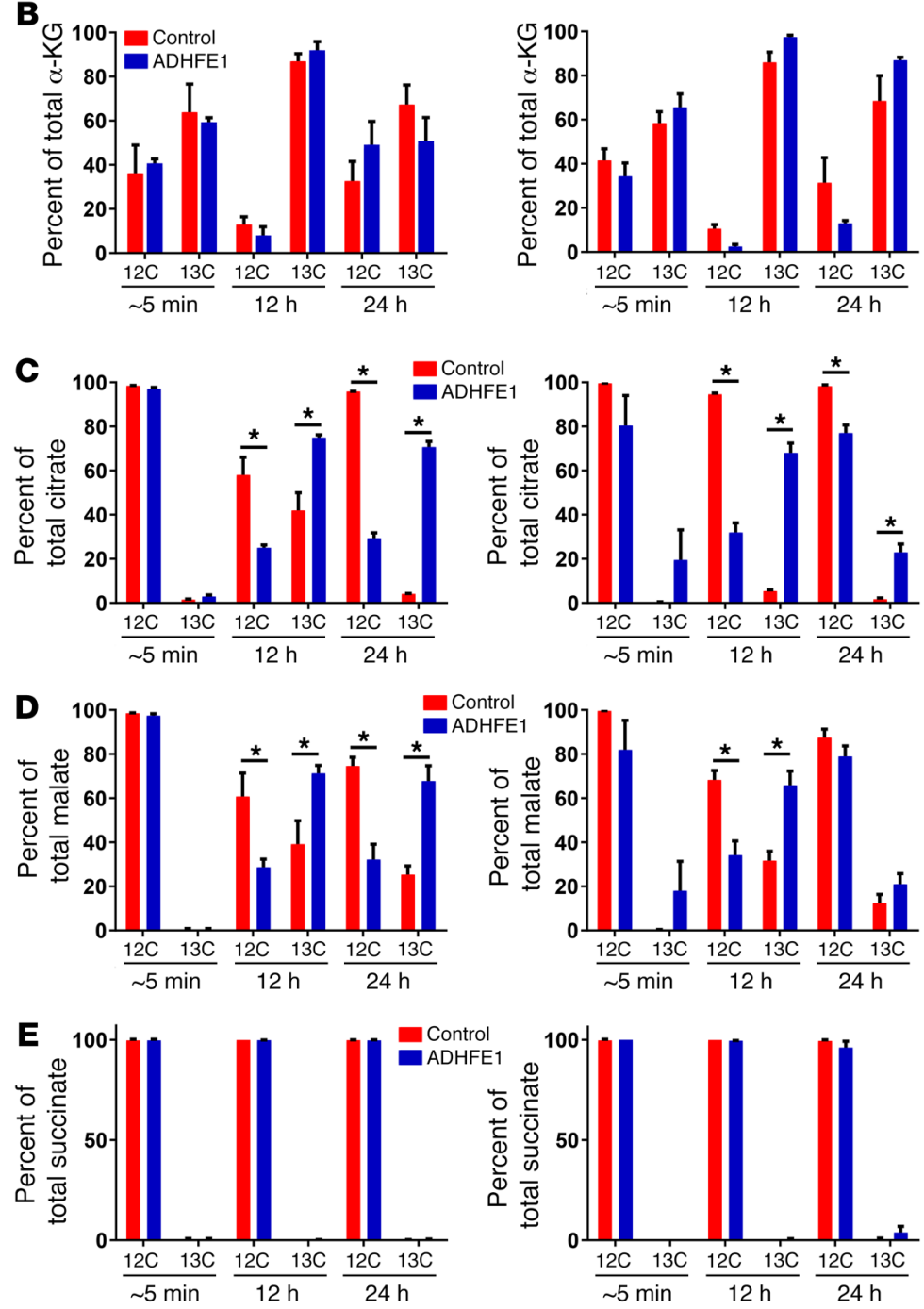

Figure 4. A retrograde tricarboxylic acid (TCA) cycle is induced by ADHFE1 in MCF10A and MCF12A cells. (A-F) Cells were cultured with $2 \mathrm{mM}\left[{ }^{\left[1-{ }^{13} \mathrm{C}\right] \text { glutamine }}\right.$ and cell extracts were prepared after approximately 5 minutes, 12 hours, and 24 hours. Incorporation of the ${ }^{13} \mathrm{C}$ label into $\alpha$-ketoglutarate ( $\alpha$-KC) (B), citrate (C), malate (D), and succinate (E) was measured by mass spectrometry. (F) Only reductive carboxylation of glutamine ("retrograde TCA") allows incorporation of $1{ }^{13} \mathrm{C}$ into citrate and malate. (C) Incorporation of all-carbon ${ }^{13} \mathrm{C}$-labeled glutamine into succinate and citrate in MCF10A cells exposed to $1 \mathrm{mM}$ cell-permeant D-2HG. m4, [1-4 $\left.{ }^{13} \mathrm{C}\right]$-labeled succinate or citrate; $\mathrm{m5}$, $\left[1-5{ }^{13} \mathrm{C}\right]$-labeled citrate. Shown is the mean \pm SD for triplicate experiments. ${ }^{*} P<0.05$ ( 2 -sided $t$ test), versus vector control or PAMO-treated cells (G).
$\mathbf{F}$

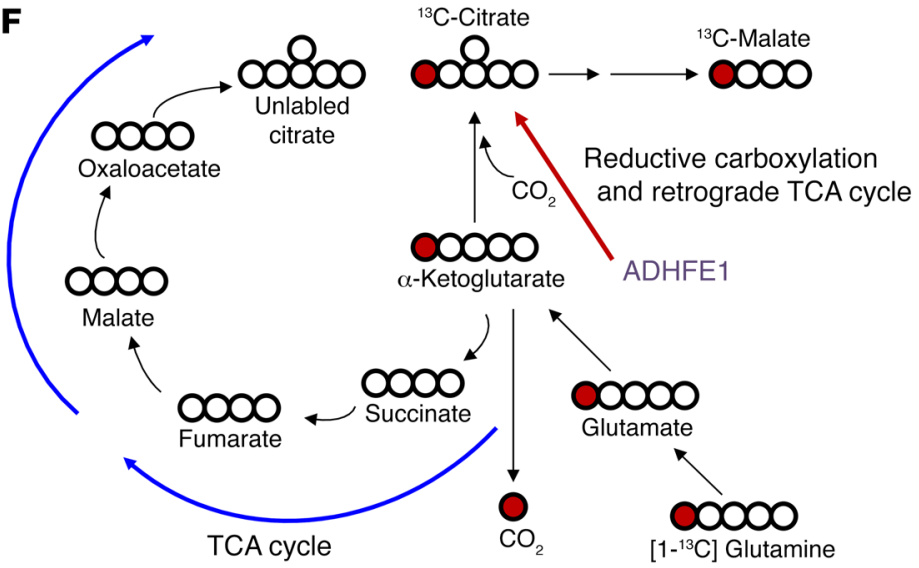

G

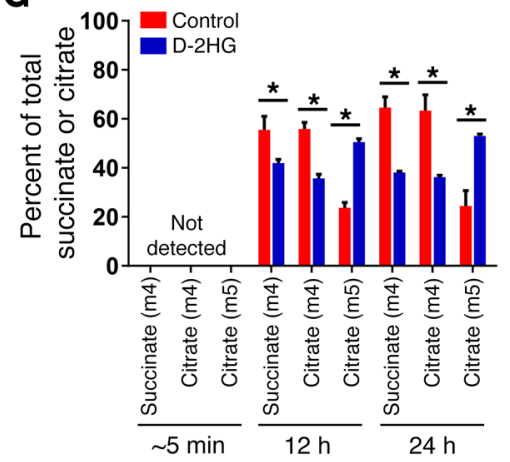


A

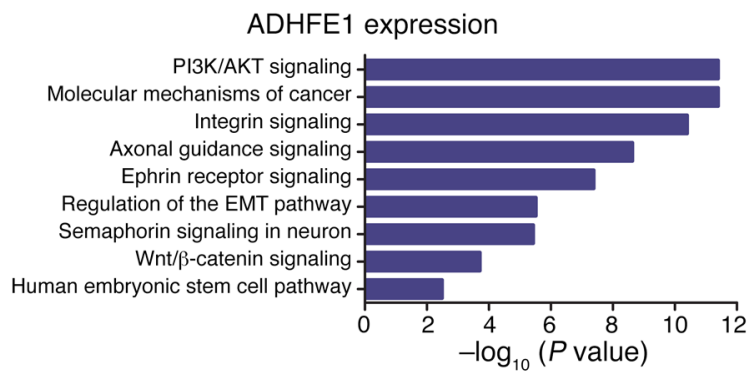

B

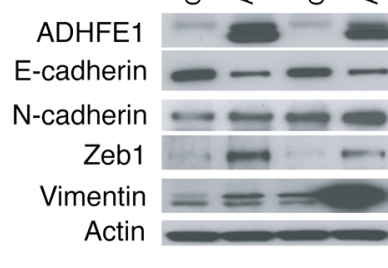

C

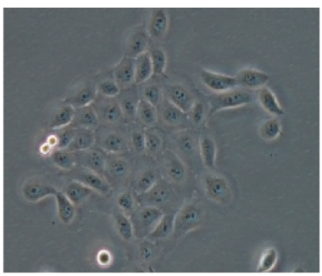

Control

MCF10A

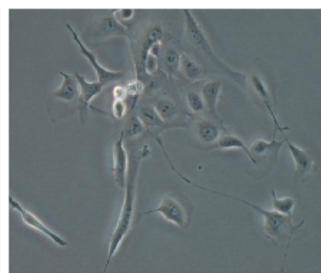

ADHFE1

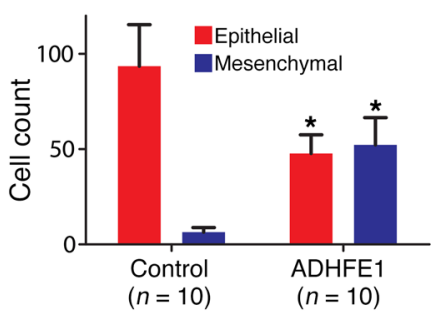

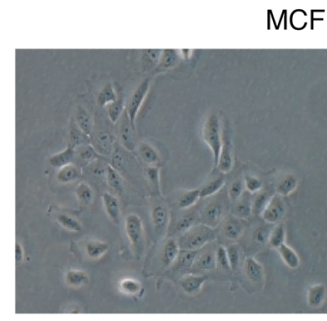

Control

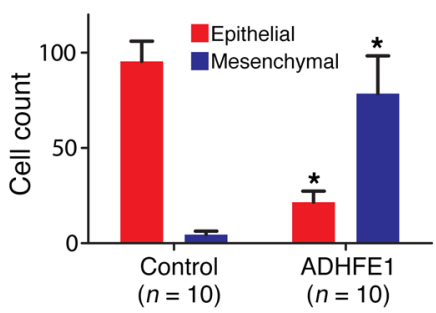

Figure 5. ADHFE1 induces epithelial-to-mesenchymal transition (EMT) and enhances invasion. (A) Ingenuity pathway analysis showing pathways that are enriched for differently expressed genes comparing MCF12A cells with the ADHFE1 transgene versus vector control cells. Pathways are ranked by $P$ values representing significance of enrichment. (B) Downregulation of E-cadherin and increased expression of mesenchymal markers in MCF10A and MCF12A cells overexpressing ADHFE1. (C) Cells with upregulated ADHFE1 develop a mesenchymal phenotype as shown by loss of cellcell adhesion and a spindle-shaped morphology. Original magnification, $\times 200$. Graphs below show quantitative analysis of this cell morphology. Shown is the mean \pm SD for 10 counted areas (per 100 cells). (D) Increased invasion of cells overexpressing ADHFE1. Invasion was examined using the $x$ Celligence system. Shown is the mean \pm SD for triplicate experiments. ${ }^{*} P<$ 0.05 , compared with control cells (2-sided $t$ test). Part of Figure 4B is shown in Figure $1 \mathrm{D}$.
D

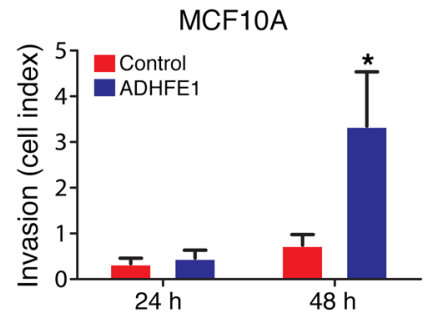

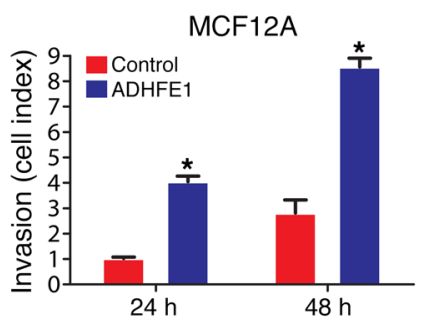

teristics comparable to octyl-2HG and also controls for octanol release. At $1 \mathrm{mM}$ concentration, octyl-2HG increases intracellular D-2HG to levels observed in breast tumors (7). These experiments showed that ADHFE1 and D-2HG similarly regulate 3 inter-related pathways, namely axon guidance, EMT, and the embryonic stem cell pathway, as indicated by the enrichment scores for differently expressed genes using Ingenuity and KEGG pathway analysis tools (Figures 5A and 6A, and Supplemental Figures 10A and 11). Like EMT, axon guidance controls cell differentiation and motility in epithelial cells but does so through a different signaling cascade $(24,25)$. To follow up on these pathway enrichment leads, we studied EMT induction and cell motility in additional cell culture experiments. Using an assessment of mesenchymal morphology and EMT markers, we confirmed that both ADHFE1 and D-2HG similarly induce EMT-like characteristics in MCF10A, MCF12A, and MCF7 cells (Figures 5 and 6, and Supplemental Figures 10 and 12). Consistent with increased mesenchymal morphology and EMT, ADHFE1 expression and D-2HG treatment augmented matrigel invasion (Figures 5 and 6) and cell migration (Supplemental Figure 13). Together, these data provide evidence that ADHFE1 and D-2HG equally induce mesenchymal transdifferentiation and an invasive phenotype in breast epithelial cells.

To further validate these cell culture-based findings, and to demonstrate their importance in human tumor biology, we examined 116 human breast tumors by immunohistochemistry (IHC) and found that ADHFE1 expression was high in 30\% (35 of 116) of the tumors and inversely correlated with E-cadherin expression in them (Figure 7A). A gene set enrichment analysis (GSEA) of TCGA gene expression data for breast cancer revealed that breast tumors harboring an ADHFE1 amplification commonly contained an EMT signature associated with a basal-like phenotype (26), when compared with breast tumors without an ADHFE1 amplification (Figure 7B). The observations also corroborate the TCGA proteome data linking ADHFE1 amplifications to increased ADHFE1 and vimentin expression and decreased E-cadherin expression in basal-like breast tumors that we referred to earlier. Together, the cell culture and tumor data provide strong evidence that ADHFE1 is an important regulator of EMT in breast cancer biology.

ADHFE1 downregulation promotes mesenchymal-to-epithelial transition and N-acetylcysteine inhibits ADHFE1-induced EMT. 
A

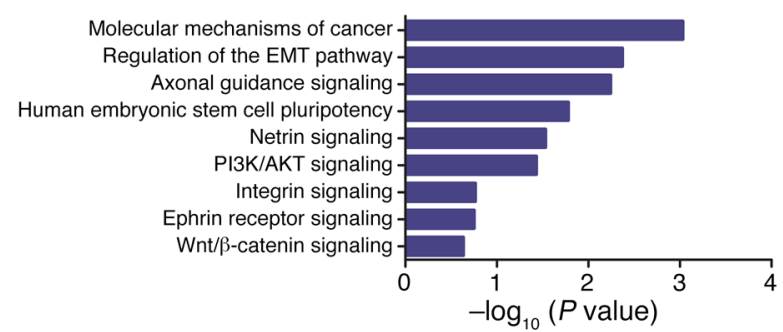

Figure 6. Cell-permeant D-2-hydroxyglutarate (octyl-2HC) induces EMT and increases invasion. (A) Ingenuity pathways enriched for genes that are differently expressed between octyl-2HG-treated (48 hours) and control MCF12A cells. (B and D) Octyl-2HG induces a mesenchymal phenotype in MCF10A and MCF12A cells. Original magnification, $\times 200$. Cell counts are shown as the mean \pm SD for 5 counted areas (per 100 cells). (C and E) Increased invasion of MCF10A and MCF12A cells that were treated with octyl-2HG. Invasion was examined using the xCelligence system. All graphs: Shown is the mean \pm SD for triplicate experiments. ${ }^{*} P<0.05$ (2-sided $t$ test) versus control cells. Cells were treated with $1 \mathrm{mM}$ octyl-2HG or $1 \mathrm{mM}$ PAMO (control).

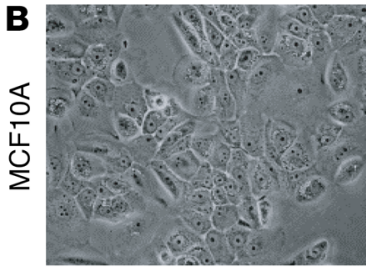

Control

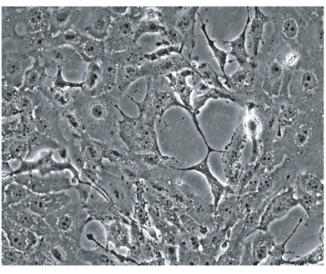

Octyl-2HG $48 \mathrm{~h}$

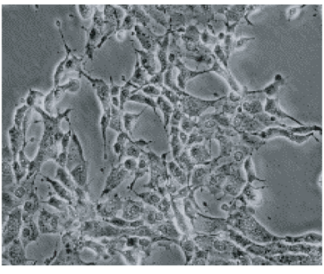

Octyl-2HG $72 \mathrm{~h}$

C

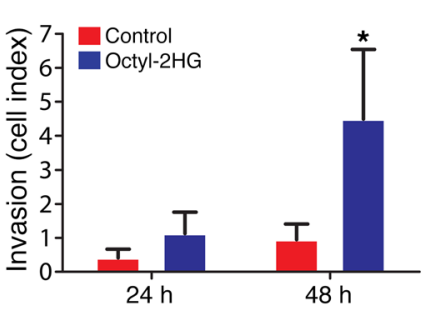

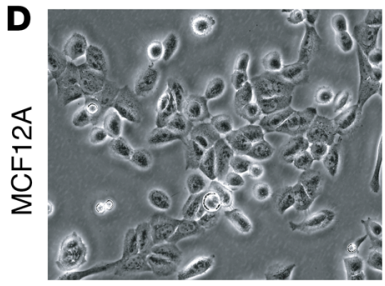

Control

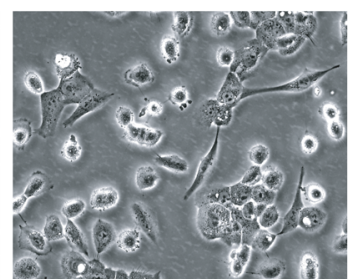

Octyl-2HG $48 \mathrm{~h}$

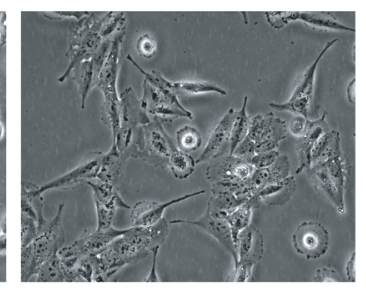

Octyl-2HG 72 h

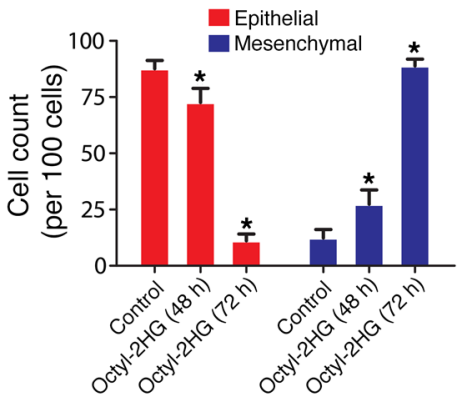

$\mathbf{E}$

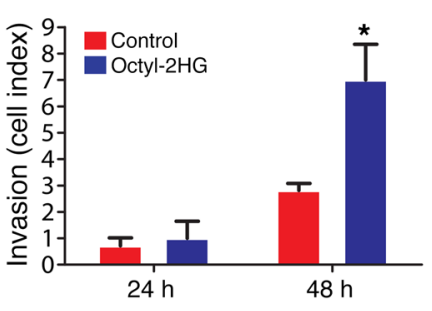

Since ADHFE1 overexpression induced EMT, we tested whether knockdown of ADHFE1 may promote mesenchymal-to-epithelial transition (MET) in a breast cancer cell line, MDA-MB-231, with mesenchymal characteristics. We downregulated ADHFE1 in these cells using siRNA and assessed MET. As shown in Figure 8A, transfection of MDA-MB-231 cells with 2 siRNAs targeting ADHFE1 led to an increase in epithelial morphology and upregulation of the epithelial marker, E-cadherin, while the expression of vimentin decreased. When ADHFE1 siRNA-transfected MDA-MB-231 cells were cocultured with cell-permeant D-2HG, the occurrence of MET due to ADHFE1 downregulation was suppressed (Figure 8A and Supplemental Figure 14).

Metabolic reprogramming during cell transformation and iron metabolism can both cause an increase in oxygen radicals that are known to induce EMT (27). Accordingly, we tested whether ROS are increased by ADHFE1, and whether ROS scavenging by the antioxidant, $N$-acetylcysteine, can prevent ADHFE1-induced EMT in MCF10A and MCF12A cells. We used a fluorochrome, MitoSOX, to quantify mitochondrial superoxide by FACS analysis. Our findings revealed a $30 \%$ to $50 \%$ increase in superoxide production in both MCF10A and MCF12A cells upon upregulation of ADHFE1 (Figure 8, B and C), indicating that ROS may contribute to ADHFE1-induced EMT. Notably, we also observed that treatment with $1 \mathrm{mM}$ cell-permeant D-2HG led to a significant increase of mitochondrial ROS in these cell lines (Supplemental Figure 15). To further verify the link between ROS and ADHFE1-induced EMT, we exposed MCF10A and MCF12A cells with upregulated ADHFE1 to $N$-acetylcysteine, 24 hours prior to the assessment of 

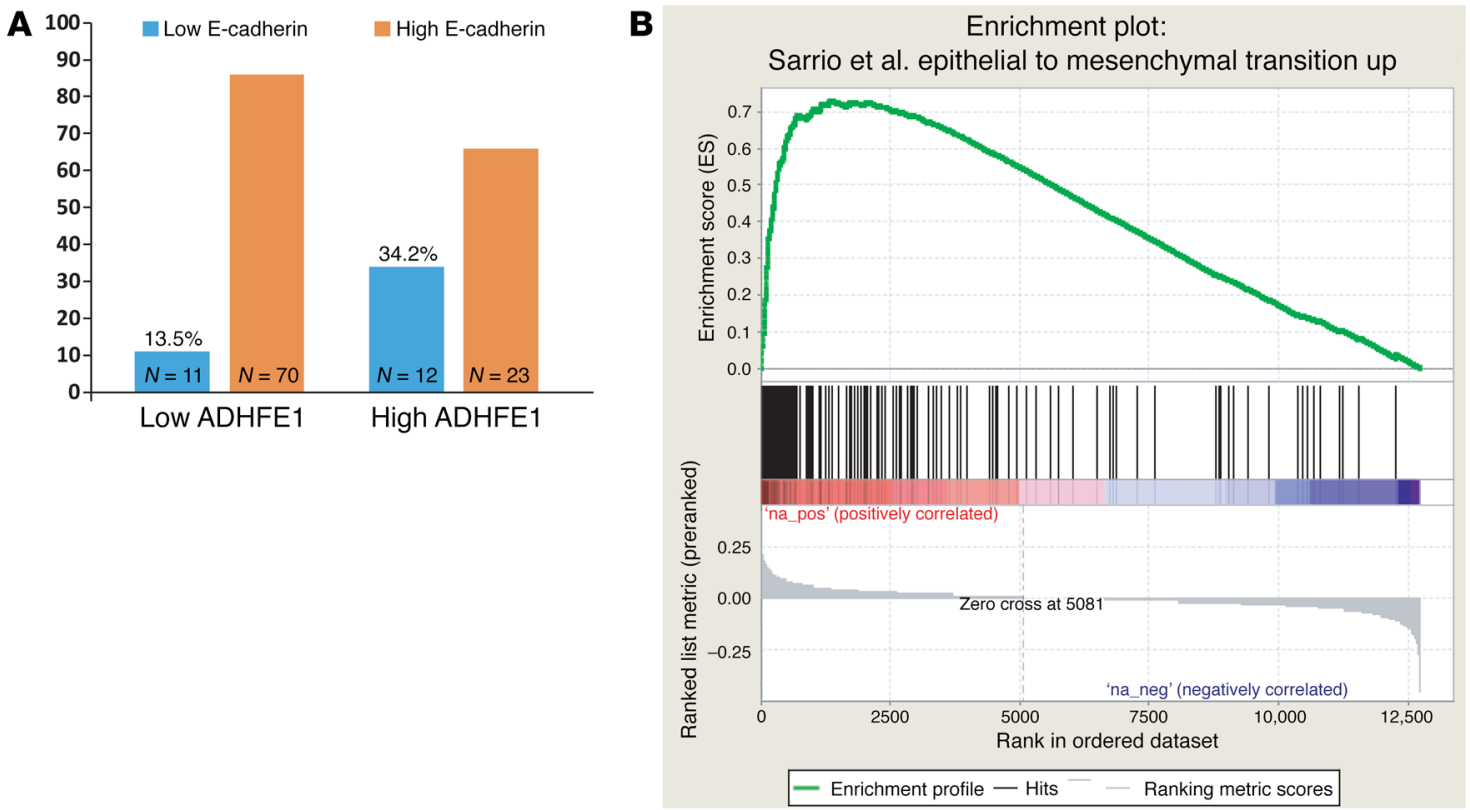

C

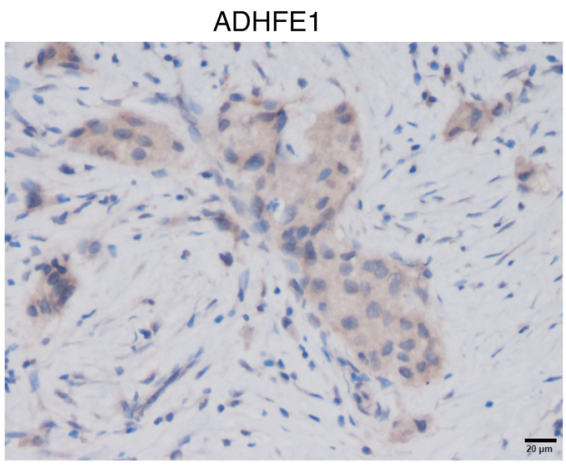

D

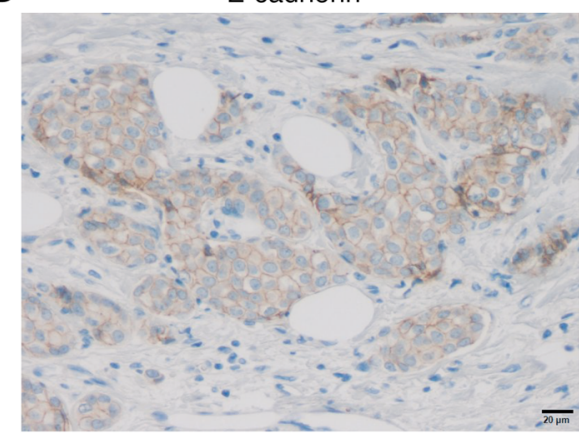

Figure 7. Reduced E-cadherin in tumors with high ADHFE1 and presence of an EMT signature in TCGA breast tumors with ADHFE1 amplifications. (A) Evaluation of ADHFE1 and E-cadherin expression in 116 human breast tumors using immunohistochemistry. Tumors with high ADHFE1 expression ( $n=$ 35) were significantly more likely to show low E-cadherin expression (34.2\%) than tumors with low ADHFE1 expression (13.5\%). $P=0.02$, Fisher's exact test. (B) Differentially expressed genes between breast tumors with and without ADHFE1 amplifications (reference) were enriched for an EMT signature. The GSEA enrichment score was high (0.7) for upregulated EMT signature genes (black bars) in tumors with amplifications. FDR $<0.001$. Forty-five breast tumors in the TCGA database had an ADHFE1 amplification. (C) Immunohistochemistry with representative ADHFE1 expression in a breast tumor. ADHFE1 is seen in the cytoplasm of the tumor epithelium (brown chromogen) and has a granular distribution, consistent with the mitochondrial location of the enzyme. (D) Representative E-cadherin expression in a breast tumor. Shown is staining of the cell membrane that is typical for this protein. Original magnification, $\times 400$ (C and D).

EMT. The data showed that prior exposure to $\mathrm{N}$-acetylcysteine inhibits the ADHFE1-induced mesenchymal transdifferentiation (Figure 8, D and E). In contrast, treatment with oxalomalate, a competitive inhibitor of $\mathrm{NADP}^{+}$-dependent IDH1 and IDH2, did not affect the expression of ADHFE1-induced EMT markers (Supplemental Figure 16). This latter observation argues against a role of reductive carboxylation in the development of EMT.

ADHFE1 and D-2HG induce mammosphere growth. Ingenuity pathway analysis (IPA) predicted that ADHFE1 and D-2HG enhance embryonic stem cell pluripotency in mammary cells and stem cell markers like $A L D H 1$ were significantly upregulated in ADHFE1-overexpressing cells. To further validate this finding, we carried out the sphere formation assay to examine whether ADHFE1 and D-2HG increase stem cell-like features in MCF10A and MCF12A cells by scoring for both sphere formation and differenti- ation into terminal ducts (Supplemental Figure 17), hypothesizing that ADHFE1 and D-2HG would increase sphere formation but inhibit terminal duct formation. As shown in Figure 9, both ADHFE1 and 2HG induced sphere formation while reducing the number of terminal ducts, indicating suppression of differentiation.

Increased histone trimethylation at histone $\mathrm{H3}$ lysine 4 in ADHFE1-overexpressing MCF7 cells. While our data suggest a substantial role of ROS in the induction of EMT by ADHFE1, additional mechanisms may contribute to mesenchymal transition, increased mobility, and dedifferentiation of breast cancer cells with upregulated ADHFE1. 2HG inhibits histone lysine demethylases, which can lead to increased methylation of histones (28, 29). It was shown that D-2HG increases trimethylation of histone $\mathrm{H} 3$ lysine $4(\mathrm{H} 3 \mathrm{~K} 4 \mathrm{me} 3)$ in the promoter region of the EMT regulator, ZEB1, in HCT116 colorectal cancer cells (30). Increased 
A Control siRnA

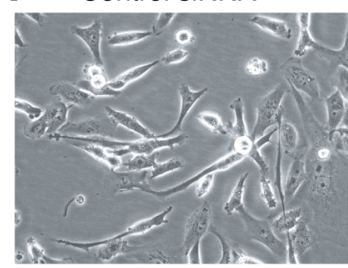

ADHFE1 SiRNA\#1
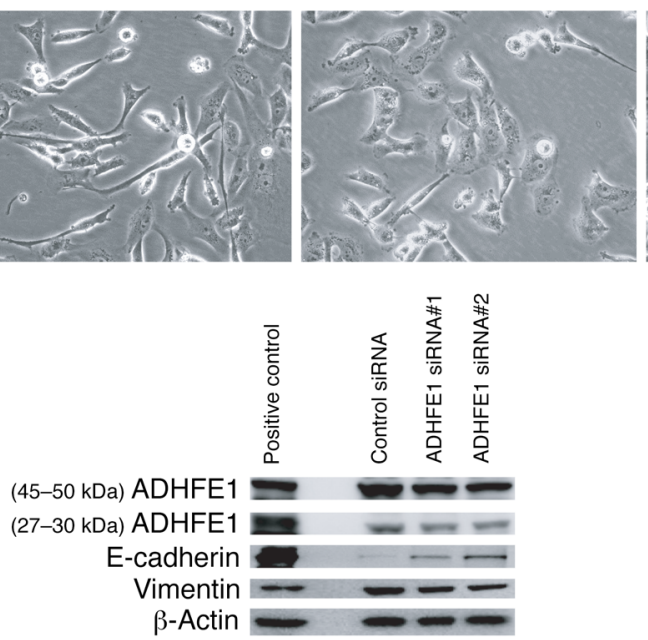

$\beta$-Actin

MCF10A

B

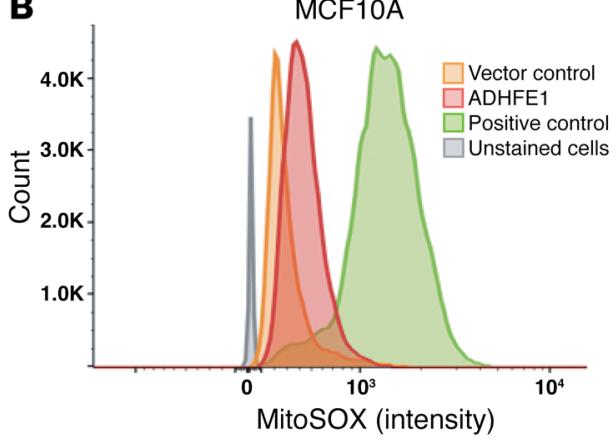

D

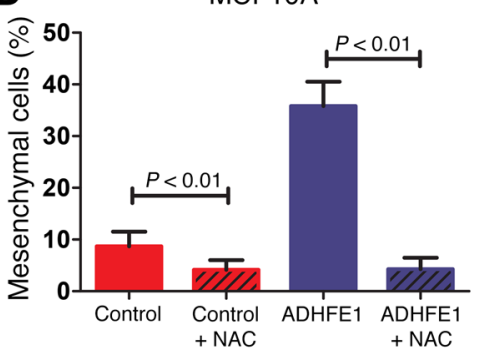

C

E
ADHFE1 SIRNA\#2
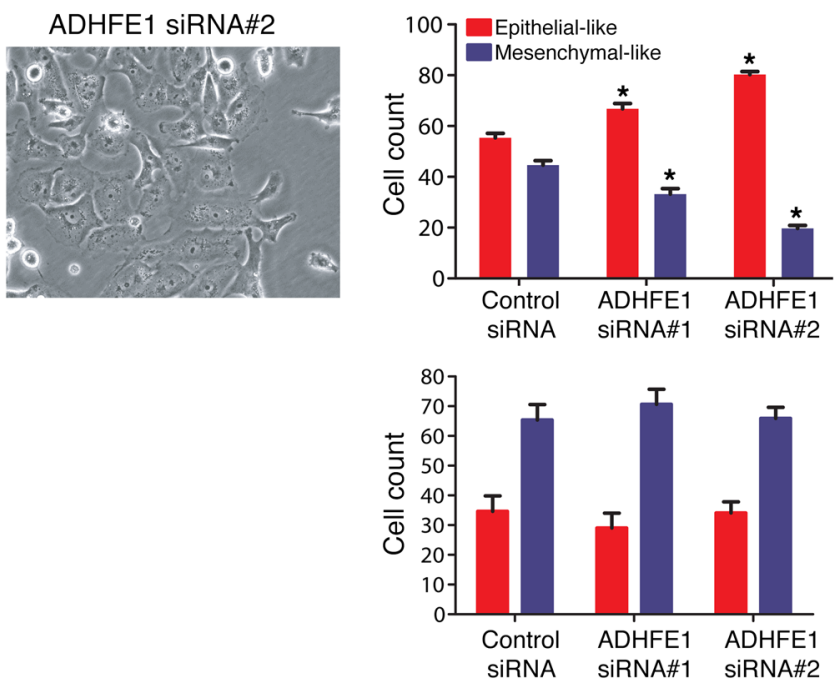

+ Cell-permeant D-2HG

MCF12A
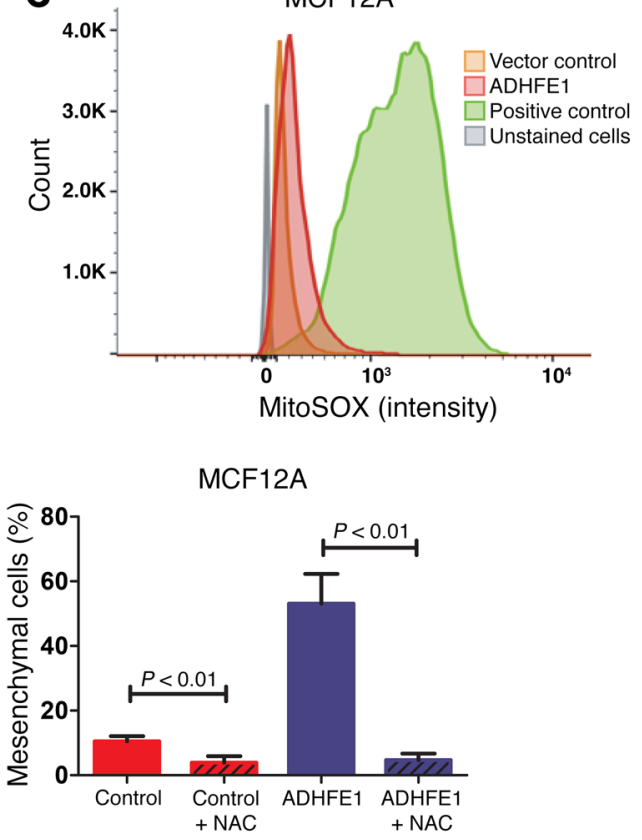

Figure 8. ADHFE1 downregulation promotes mesenchymal-to-epithelial transition (MET) and $\mathbf{N}$-acetylcysteine inhibits ADHFE1-induced EMT. (A) Increase in epithelial characteristics in MDA-MB-231 cells after downregulation of ADHFE1 with siRNA. Addition of cell-permeant D-2HG inhibits siRNAinduced MET. ${ }^{*} P<0.01$ versus control siRNA. Shown is the mean \pm SD for 10 counted areas (per 100 cells) and protein expression at day 3 after siRNA transfection. Original magnification, $\times 200$. (B and C) The mitochondrial superoxide indicator, MitoSOX, shows increased superoxide production in MCF10A and MCF12A cells with upregulated ADHFE1. Shown are representative flow cytometry experiments. MCF10A cells, $27.3 \% \pm 19.6 \%$ superoxide increase versus vector control $(n=4)$; MCF12A cells, $48.2 \% \pm 5.5 \%(n=4) ; P<0.05$. (D and E) Inhibition of ADHFE1-induced EMT by $N$-acetylcysteine (NAC). MCF10A and MCF12A cells were cultured for 3 days. NAC $(10 \mathrm{mM})$ was added 24 hours prior to assessment of EMT. Number of cells with mesenchymal features was counted in 5 representative areas as percentage of all cells. Significance of differences in A, D, and $\mathbf{E}$ was determined with a 2-sided $t$ test.

H3K4me3 near transcriptional start sites is a marker of increased transcription (31). Therefore, we performed a genome-wide ChIPseq analysis for $\mathrm{H} 3 \mathrm{~K} 4 \mathrm{me} 3$ binding to gene loci in ADHFE1overexpressing MCF7 cells and compared their H3K4me3 profile to vector control cells and MCF7 cells overexpressing MYC. The analysis showed that both ADHFE1- and MYC-overexpressing cells have increased H3K4me3 (Supplemental Figure 18). H3K4me3 was globally increased in promoter regions of genes regulating EMT, axon guidance, and stemness (Figure 10, A-C), indicating activation of these pathways. However, H3K4me3 was not significantly increased in the ZEB1 promoter region. Instead, other genes showed more striking increases in H3K4me3 near the transcription start site, such as the mesenchymal marker $\mathrm{N}$-cadherin, the axon guidance pathway markers SEMA3A and SEMA3D, and the stem cell markers SOX9 and FGFR2, as examples. FGFR2 has important roles in embryonic development and is a breast cancer susceptibility gene (32). SOX9 is an embryonic transcription factor and key regulator of the mammary stem cell 
A

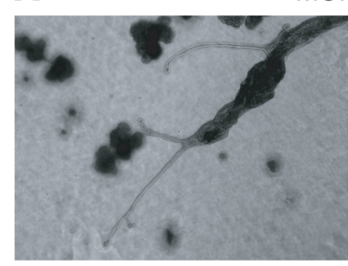

Control

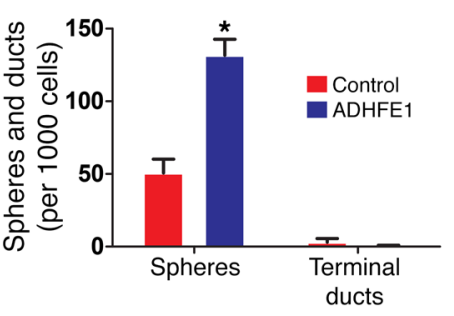

B

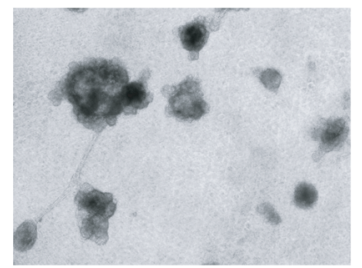

Control
MCF10A

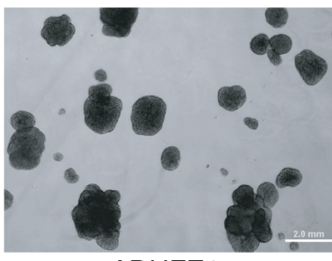

ADHFE1

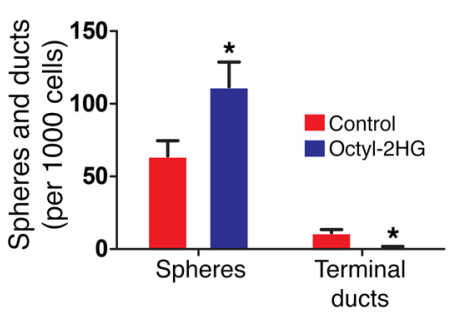

MCF12A
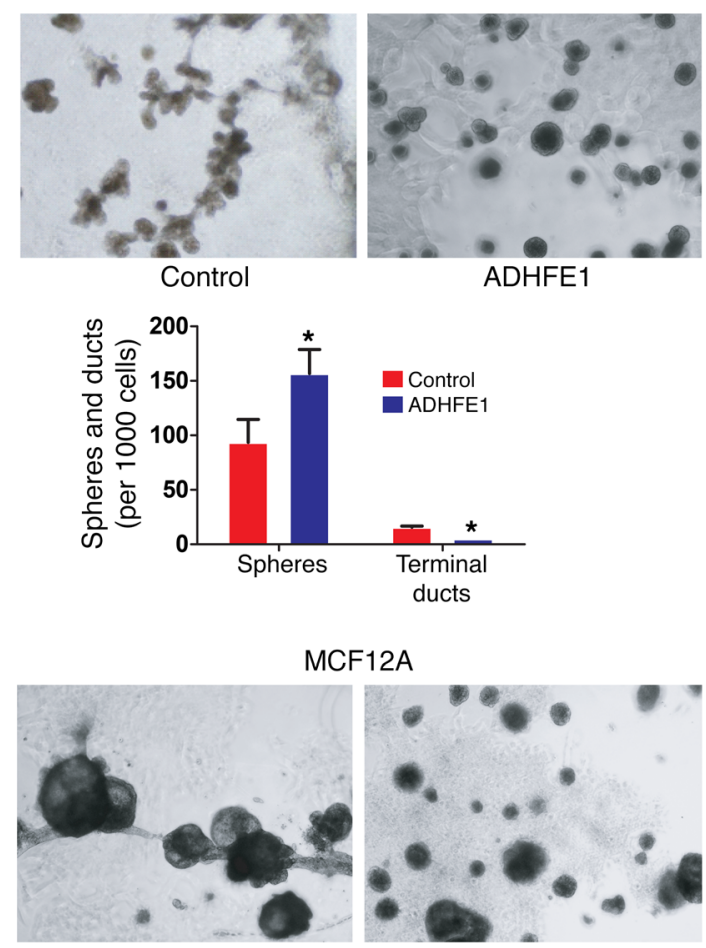

Control

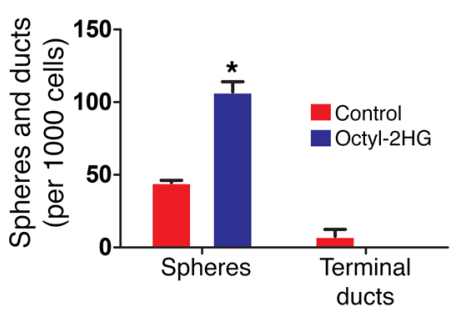

Figure 9. ADHFE1 and cell-permeant D-2-hydroxyglutarate induce sphere formation of MCF10A and MCF12A cells. (A) Upregulated ADHFE1 induces sphere formation and inhibits differentiation into terminal ducts in MCF12A cells. Control shows terminal ducts. Original magnification, $\times 40$. (B) Cellpermeant 2-hydroxyglutarate (octyl-2HG) induces sphere formation and inhibits differentiation into terminal ducts. Original magnification, $\times 40$. In A and B, data are the mean \pm SD for triplicate experiments. ${ }^{*} P<0.05$ versus control cells ( 2 -sided $t$ test). Cells were treated with 1 mM octyl-2HC or 1 mM PAMO (control). In A, terminal duct numbers for MCF10A cells were too low at baseline to be scored.

state that promotes metastasis-seeding abilities, Wnt signaling, and breast cancer endocrine resistance (33-35). ADHFE1 may also affect the DNA methylation state of cells, as $2 \mathrm{HG}$ is an inhibitor of 5-methylcytosine hydroxylases (36). Accordingly, we found that ADHFE1 altered DNA methylation in loci encoding the EMT markers E-cadherin, vimentin, and N-cadherin in MCF12A cells (Supplemental Figure 19), suggesting that ADHFE1 may increase EMT and other pathways in various ways including ROS production and its effects on histone and DNA methylation (Figure 10D).

\section{Discussion}

In this study, we show that D-2HG-producing ADHFE1 is a breast cancer oncogene that is associated with an aggressive disease. Furthermore, our study shows that ADHFE1 promotes metabolic reprogramming and a reductive glutamine metabolism with increased D-2HG and ROS formation, leading to cellular dedifferentiation and mesenchymal transition, like alterations that occur in cells with IDH mutations $(37,38)$. We also show that D-2HG by itself induces these phenotypes.

Accumulation of $2 \mathrm{HG}$ has been reported for different cancer types. In gliomas and myeloid leukemia, D-2HG accumulates in tumors because of IDH mutations $(9,10)$. However, these mutations are rare in breast cancer (39). Instead, $2 \mathrm{HG}$ accumulates in breast tumors in association with MYC signaling (7). Conversely, L-2HG is the predominant enantiomer that increases in renal tumors because of a downregulation of the L-2HG dehydrogenase in them (40). Other studies showed that L-2HG accumulates under hypoxia by different mechanisms $(41,42)$. Thus, hypoxia may increase $2 \mathrm{HG}$ levels in breast tumors. We found that ADHFE1 upregulates intracellular D-2HG under hypoxic conditions, reaching a 5- to 15 -fold increase over baseline in cell culture experiments, providing a potentially new mechanism of D-2HG accumulation in breast cancer. We also observed that L-2HG increased in breast epithelial cells that were cultured under hypoxia (data 
A Epithelial-mesenchymal transition
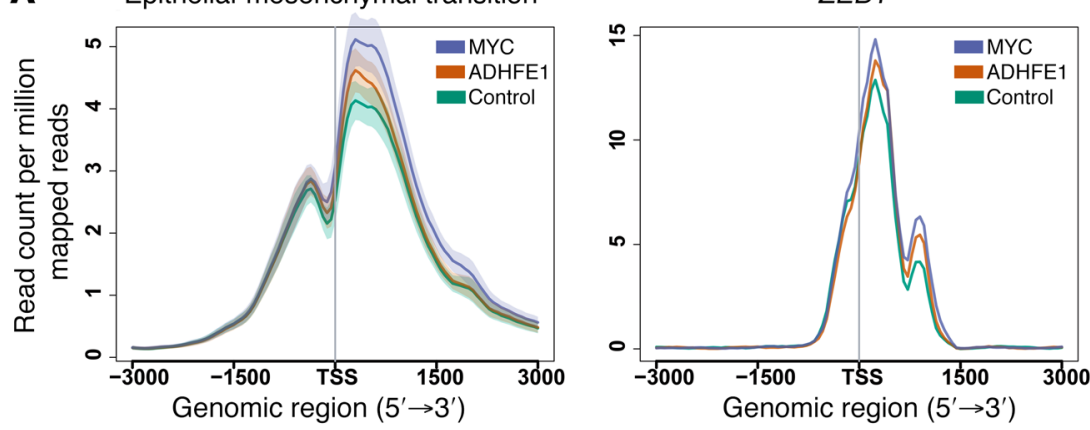

$\mathrm{CDH} 2$
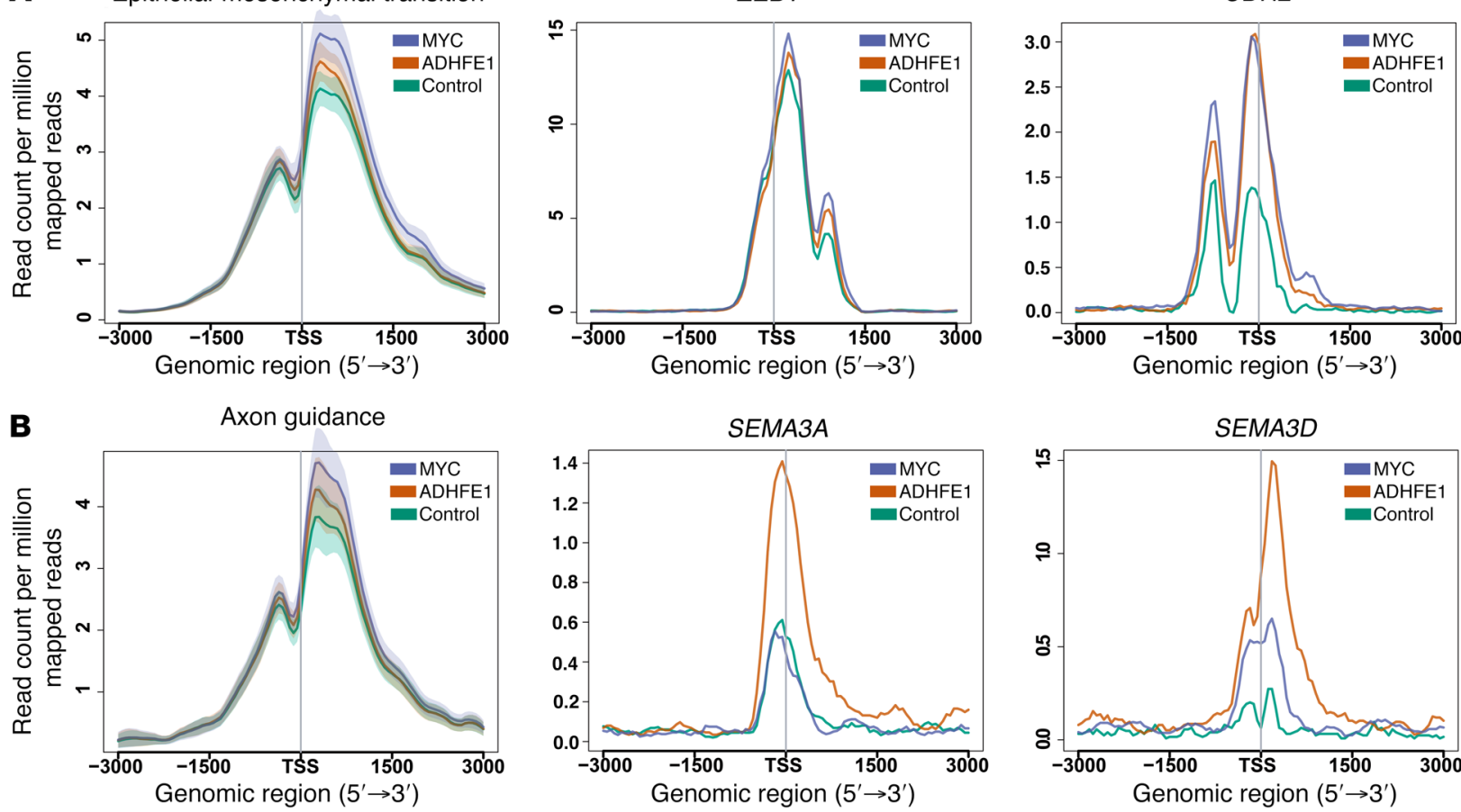

C Cignaling pathways regulating
pluripotency of stem cells
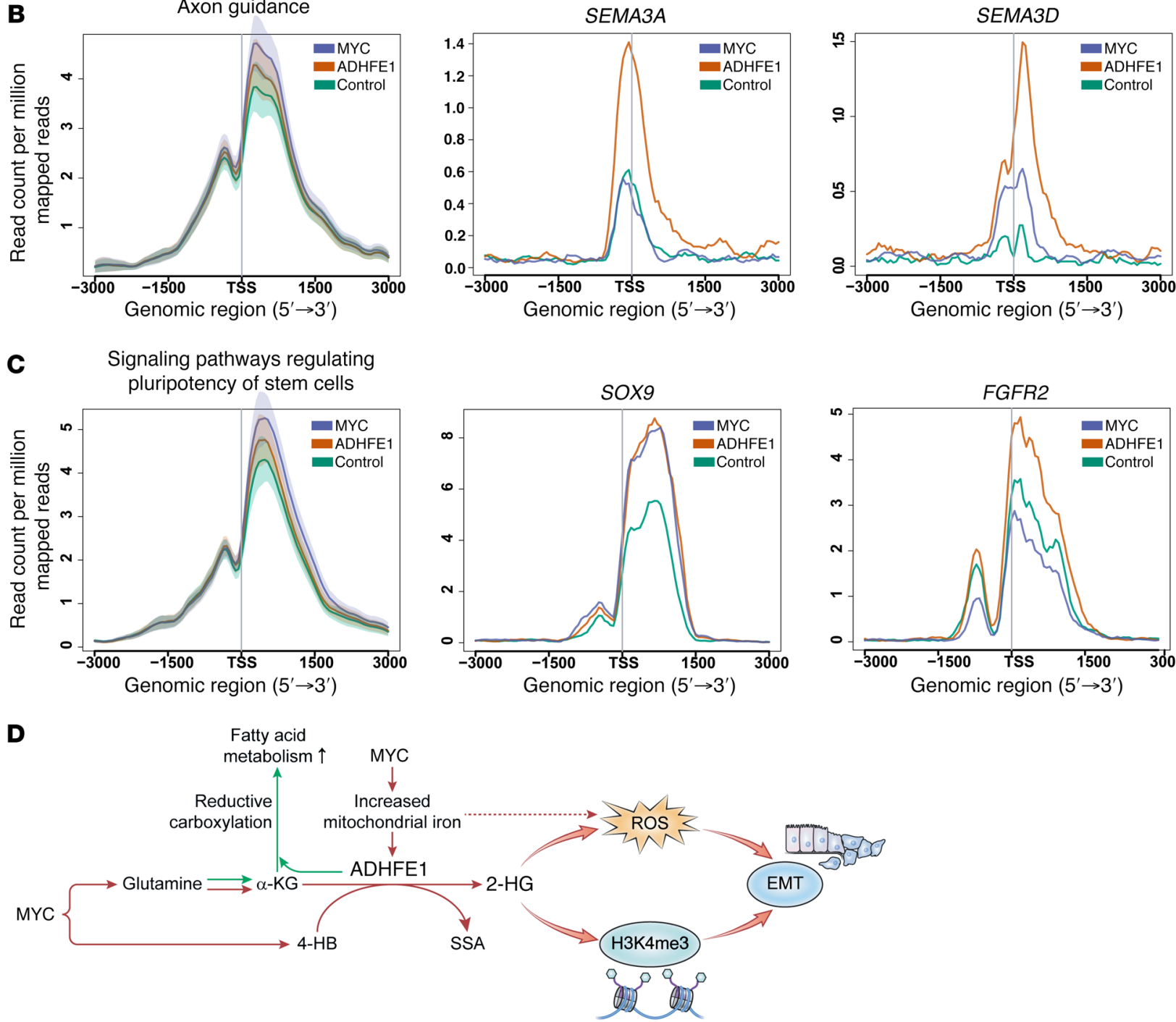

Figure 10. ChIP-seq analysis of H3K4me3 in ADHFE1- and MYC-overexpressing MCF7 cells. Trimethylation of histone H3 lysine 4 (H3K4me3) is globally increased in promoter regions of genes regulating epithelial-to-mesenchymal transition (EMT) (A), the axon guidance pathway (B), and stemness (C) in MCF7 cells overexpressing either MYC or ADHFE1 (FDR < 5\% for each pathway, compared with vector control cells). Examples for EMT pathway, ZEB1 and $\mathrm{CDH} 2$ (N-cadherin); axon guidance, SEMA3A and SEMA3D; and stem cell markers, SOX9 and FGFR2. H3K4me3 is significantly increased in the promoter region for each of the shown genes, with the exception of ZEB1, comparing ADHFE1-overexpressing versus vector control cells (FDR < 5\%). EMT pathway: 344 genes. Axon guidance pathway: 127 genes. Pluripotency of stem cells pathway: 138 genes. Shown is the summarized read coverage for triplicate experiments of the ChIP-seq data. TSS, transcription start site. (D) Upregulation of ADHFE1 and D-2HG formation in breast tumors and their relationship to EMT and metabolic reprogramming. MYC increases D-2HG formation by increasing both substrate and iron availability for the iron-containing enzyme, ADHFE1. ADHFE1 and D-2HG induce a reductive glutamine metabolism, ROS, and histone methylation at H3K4me3, leading to increased acetyl-CoA and fatty acid synthesis and EMT. $\alpha$-KG, $\alpha$-ketoglutarate; 4-HB, 4-hydroxybutyrate; SSA, succinic semialdehyde. 
not shown), and noticed that a subset of human breast tumors showed a significant increase in L-2HG. However, we did not find that L-2HG levels correlated with transcript expression of hypoxiainducible lactate dehydrogenase $\mathrm{A}$ in these tumors, an enzyme that has been linked to hypoxia-related L-2HG production (41). Instead, L-2HG strongly correlated with lactate dehydrogenase B mRNA levels $(\rho=0.93, P<0.01)$, which is an enzyme critical for the development of triple-negative tumors (43).

While there are numerous reports studying the mechanisms of IDH-dependent D-2HG accumulation in mammalian cells, few explored aberrant D-2HG accumulation in the absence of mutant IDH $(30,44,45)$. In one study, it was shown that phosphoglycerate dehydrogenase (PHGDH) produces D-2HG (44), although the reported increase in D-2HG in breast cancer cell lines due to PHGDH was modest (2- to 3-fold), and by itself would not explain a 10to 100 -fold accumulation of D-2HG in some breast tumors. In our research, we focused on the function of ADHFE1 as an oncogene and producer of $\mathrm{D}-2 \mathrm{HG}$ because this enzyme is induced by MYC and redirects glutamine metabolism, which is consistent with our tumor data linking MYC signaling and glutamine metabolism to increased $2 \mathrm{HG}$ in breast cancer (7). In our previous report, however, we did not find that ADHFE1 transcript levels correlated with $2 \mathrm{HG}$ levels. The activity of many metabolic enzymes is not tightly regulated at the expression level. Instead, the enzymatic activity can simply be regulated by the availability of its substrates, such as the availability of 4-hydroxybutyrate for ADHFE1 (17). Accordingly, we found that 4-hydroxybutyrate levels were significantly elevated in a subset of ER-negative breast tumors and correlated with $2 \mathrm{HG}$ levels in these tumors and the MCF7 mouse xenografts. 4-Hydroxybutyrate levels also correlated with the presence of the MYC signaling signature in human breast tumors (Pearson's correlation $=0.49 ; P<0.01)$ and increased in MCF7 xenograft tumors with MYC overexpression, indicating that MYC signaling may provide an added substrate availability for this enzyme in breast tumors. Fittingly, we found that MYC induces AKR7A2, a key enzyme for the synthesis of 4-hydoxybutyrate (20). As such, there can be coregulation of the D-2HG and 4-hydroxybutyrate pathways in breast tissue.

ADHFE1 promoted a reverse TCA cycle via reductive carboxylation and a moderate but significant increase in ROS production. The latter, but not reductive carboxylation, had an important role in the induction of EMT by ADHFE1. Several cellular conditions have been linked to hypoxia-induced reductive carboxylation such as high NADPH to NADP ratio and low citrate (46). ADHFE1 induced an increase in the NADPH to NADP ratio, but it did not induce depletion of citrate. Reductive carboxylation of glutamine-derived $\alpha$-ketoglutarate is an important survival mechanism in mammalian cells and constitutes a pathway to maintain citrate synthesis and lipogenesis in oxygen-deprived cells $(21,22,47,48)$. In contrast, cells expressing mutant IDH are no longer capable of performing reductive carboxylation and exhibit an increased dependence on oxidative mitochondrial metabolism $(49,50)$. Hence, cells that accumulate D-2HG using an alternative pathway, like ADHFE1, may have a survival advantage under hypoxic conditions because of their continued ability to use reductive carboxylation for acetyl-CoA synthesis. Accordingly, we found that acetyl-CoA levels are significantly increased in ADHFE1-overex- pressing MCF12A cells, and show that these cells have a growth advantage under hypoxic, but not normoxic conditions, when compared with vector control cells.

A key finding of our study is the discovery that both ADHFE1 and D-2HG similarly induce EMT, stemness, and invasion of mammary cells. It has previously been shown that mutant IDH and D-2HG promote EMT in human colon cancer cells $(30,37)$. Similarly, accumulation of fumarate in fumarate hydratase-deficient cells induces EMT through inhibition of a Tet-mediated demethylation process in the regulatory region of the miR-200ba429 cluster (51). We show that ADHFE1 and D-2HG have similar EMT-inducing effects in both breast cancer cells and benign breast epithelial cells. Increased D-2HG levels in tumors due to $I D H$ mutations inhibit $\alpha$-ketoglutarate-dependent dioxygenases (28), and through this mechanism induce various perturbations including DNA and histone methylation and a dedifferentiated cellular state (36). Our study shows that ADHFE1 promotes similar perturbations including increased $\mathrm{H} 3 \mathrm{~K} 4 \mathrm{me} 3$, a marker for active transcription, in the promoter regions of genes related to mesenchymal transition and stem cell pluripotency. As such, ADHFE1 induces many of the same phenotypes in breast cancer cells that have been described for mutant IDH-derived D-2HG but are independent of IDH mutations. While studies have shown that D-2HG can significantly increase cell proliferation in leukemia under normoxic conditions (52), we did not find that D-2HG enhances normoxic growth of MCF10A and MCF12A cells but rather their invasiveness, which may constitute a difference between breast epithelial cells and other cells. Lastly, the effects of D-2HG as an oncometabolite have been attributed to its intracellular accumulation. Notably, our data show that ADHFE1-overexpressing cells efficiently secrete D-2HG into the extracellular space, potentially affecting the tumor microenvironment.

ADHFE1 is an iron-sulfur enzyme and a member of the metaldependent alcohol dehydrogenase family and may have an important function in fatty acid metabolism $(17,53)$. Its expression is likely linked to iron metabolism (16). In our study, we show that $\mathrm{Fe}^{2+}$ upregulates ADHFE1 expression in mammary epithelial cells. We also show that upregulation of ADHFE1 by MYC can be inhibited by a chelator of $\mathrm{Fe}^{2+}$. MYC is a key regulator of iron metabolism and upregulates genes in this pathway (15). An increased expression of iron metabolism genes in breast cancer cell lines with an aggressive mesenchymal phenotype has been described (54) and may indicate increased MYC signaling. We did not find that MYC induces ADHFE1 through a direct mechanism, suggesting that the effect of MYC on iron metabolism is a primary driver of MYC-induced ADHFE1. Together, our data raise the possibility that $2 \mathrm{HG}$ accumulation and iron metabolism are linked in breast cancer, while iron metabolism may also account for at least some of the excessive ROS formation that we observed in ADHFE1overexpressing cells.

In conclusion, our study shows that human breast tumors produce both D-2HG and L-2HG but predominately accumulate D-2HG. Moreover, we nominate the MYC-linked mitochondrial enzyme, ADHFE1, as a candidate breast cancer oncogene that induces a reductive glutamine metabolism in mammary cells and increases D-2HG production. Lastly, we link D-2HG to mesenchymal reprogramming and enhanced invasion. Together, our data 
support the hypothesis that ADHFE1 and MYC signaling contribute to the accumulation of D-2HG in breast tumors and show that $\mathrm{D}-2 \mathrm{HG}$ is an oncogenic metabolite and potential driver of disease progression at levels that can occur in these tumors.

\section{Methods}

Reagents and lentiviral vectors. Octyl-D-2HG ([2R]-2-hydroxyglutaric acid octyl ester), abbreviated as octyl-2HG in the text, and control compound PAMO were custom synthesized by SLR Biosciences and were added to cell culture medium in DMSO as solvent $(1 \mu \mathrm{l}$ per $2 \mathrm{ml}$ culture medium). PAMO has cell permeability characteristics similar to $2 \mathrm{HG}$ and is also a control for octanol release. Additional information about reagents can be found in the Supplemental Methods.

Human tissues. We analyzed human breast tumors for tissue levels of D- and L-2-hydroxyglutarate. Collection of these tissues has previously been described $(7,12)$.

Cell lines. Human nontumorigenic and tumorigenic breast epithelial cell lines, MCF10A, MCF12A, MCF7, and MDA-MB-231, were obtained from American Type Culture Collection. MCF10A and MCF12A cells were cultured in DMEM/F12 (1:1) (Invitrogen/Thermo Fisher Scientific) supplemented with $5 \%$ heat-inactivated horse serum (Invitrogen), $500 \mathrm{ng} / \mathrm{ml}$ hydrocortisone (MilliporeSigma), $10 \mu \mathrm{g} / \mathrm{ml}$ insulin (Invitrogen), $100 \mathrm{ng} / \mathrm{ml}$ cholera toxin (MilliporeSigma), 20 ng/ml EGF (Invitrogen), and 1\% antibiotics. MCF7 cells were grown in RPMI (Invitrogen) supplemented with 10\% FBS (Invitrogen), and $1 \%$ antibiotics (Invitrogen). MDA-MB-231 cells were grown in DMEM (Invitrogen) with $2 \mathrm{mM}$ glutamine (MilliporeSigma), 10\% FBS (Invitrogen) and 1\% antibiotics (Invitrogen). Authentication of these cell lines was performed with a short tandem repeat analysis with GenePrint10 (9 loci + amelogenin for sex determination).

ADHFE1 overexpression after lentiviral infection. To obtain stable cell lines overexpressing ADHFE1, we infected MCF7, MCF10A, MCF12A, cells with lentiviruses containing pFUGW-CMV51p $>$ Hs. ADHFE1-FLAG and selected with puromycin $(2 \mu \mathrm{g} / \mathrm{ml})$ to obtain stable clones. Control cells were obtained after selection of cells with puromycin that contained the pFUGW-CMV51p control vector.

ADHFE1 knockdown with siRNA. Transfection with siRNA targeting ADHFE1 was performed as previously described (7).

Inducible expression/knockdown of MYC in HMEC and Sum159T cells. HMECs with an inducible $M Y C$-estrogen receptor fusion transgene (HMEC-MYC) were cultured as described previously $(7,13)$. Cells were cultured in mammary epithelial growth medium (MEGM; Lonza), and MYC was induced with $300 \mathrm{nM}$ 4-hydroxytamoxifen (for 48 hours) and harvested. In these cells, which do not endogenously express the ER, addition of tamoxifen leads to increased translocation of the MYC-ER fusion protein into the nucleus and MYC signaling. SUM-159T breast cancer cells were infected with pINDUCER lentiviral constructs containing a doxycycline-inducible shRNA targeting $M Y C$, as described previously (7). Cells were sorted for GFP to generate fully infected populations. Doxycycline $(1 \mu \mathrm{g} / \mathrm{ml})$ was added to the culture medium for 3 days to induce knockdown of MYC.

Orthotopic injection of MCF7 cells into the mammary fat pad to study tumor growth. MCF7 cells were infected with lentiviral constructs to obtain constitutive expression of the target genes, ADHFE1, MYC, or both ADHFE1 and MYC. To obtain stable cell lines overexpressing MYC, MCF7 cells with or without the ADHFE1 transgene were infected with lentivirus containing the expression vector pCMV>
Hs.MYC_IRES-eGFP and selected with neomycin $(1,600 \mu \mathrm{g} / \mathrm{ml})$, which led to a 4- to 5-fold upregulation of MYC in the selected cells (Supplemental Figure 20). Cells were inoculated into nonobese diabetic/severe combined immunodeficiency mice (NOD/SCID gamma, NOD.Cg-Prkd scid Il2rg $g^{\text {tmlWjl }} /$ SzJ, strain 005557, The Jackson Laboratory), as these mice do not require implantation of estrogen pellets for tumor growth of MCF7 cells. Additional information can be found in the Supplemental Methods.

Induction of ADHFE1 with iron supplementation. HMECs were treated with 0, 0.1, 0.5, and $1 \mathrm{mM} \mathrm{FeSO}_{4}$ (Acros Organics/Thermo Fisher Scientific), which was added to the culture medium for 24 hours. In some experiments, the iron $\left(\mathrm{Fe}^{2+}\right)$ chelator 3-AP was added to the culture medium at a $0.5 \mu \mathrm{M}$ final concentration prior to the addition of $\mathrm{FeSO}_{4}$.

ADHFE1 reporter assay. A 5-kb segment of the human ADHFE1 promoter region upstream of the transcription start site was subcloned into a GFP reporter construct (ADHFE1p $5 \mathrm{~kb}>\mathrm{Kz}$-eGFP) by the Protein Expression Laboratory, Frederick National Laboratory for Cancer Research (Frederick, Maryland, USA). The putative MYC binding site at chr8: 67341000 was mutated (ADHFE1p 5kb A507V R508P $>$ KzeGFP), which was verified by sequencing, to obtain reporter constructs for the ADHFE1 promoter with or without the MYC binding site. For the reporter assay, HMEC-MYC cells were seeded in 6-well plates $(1 \times$ $10^{6}$ per well) and transfected with either ADHFE1 wild-type or mutant MYC binding site promoters using Lipofectamine 2000 according to the manufacturer's protocol (Life Technologies) and MYC expression was induced with $300 \mathrm{nM} 4$-hydroxytamoxifen. After 48 hours, cells were washed twice with PBS, trypsinized, and transferred to polystyrene tubes for measurement of GFP at 488-nm excitation using flow cytometry (FACSCanto II Bioanalyzer, BD Biosciences). Data analysis was performed using FlowJo version 8.5.2 (Tree Star). As a positive control, we transfected the cells with the pMyc-Luc reporter from Signosis.

RNA isolation and quantitative real-time PCR measurements. Total RNA was extracted using the TRIzol/chloroform method. RNA was quantified using the Nanodrop 1000 spectrometer (Thermo Fisher Scientific). The High-Capacity cDNA Reverse Transcription Kit (Thermo Fisher Scientific) was used to create $20 \mathrm{ng} / \mu \mathrm{l}$ cDNA stock. Quantitative real-time PCR (qRT-PCR) assays were performed in triplicate using the TaqMan expression assays for ADHFE1, E-cadherin (CDH1), $\mathrm{N}$-cadherin (CDH2), or vimentin (VIM) (Applied Biosystems), which included preoptimized probes and primer sets for these genes. Data were collected using the ABI PRISM 7900HT Fast Real-Time PCR System with a 384-Well Block Module. The 18S rRNA was used as the internal standard reference. Normalized expression was calculated using the comparative $\mathrm{C}_{\mathrm{t}}$ method and fold changes were derived from the $2^{-\Delta \Delta \mathrm{Ct}}$ values for each gene.

RNA sequencing of MCF7 and MCF12A cells overexpressing ADHFE1 or treated with octyl-2HG. RNA sequencing (RNAseq) was performed in triplicate for each experimental group (MCF7 \pm ADHFE1; MCF12A \pm ADHFE1; MCF7 \pm octyl-2HG; MCF12A \pm octyl-2HG). Cells were cultured with $1 \mathrm{mM}$ octyl-2HG for 48 hours before RNA was isolated. Integrity of isolated RNA was evaluated with the Agilent 2100 Bioanalyzer (Agilent Technologies). RNAseq was performed at the Sequencing Facility, Leidos Biomedical Research, Inc., Frederick National Laboratory for Cancer Research. Briefly, $500 \mathrm{ng}$ of total RNA was used for library preparation with the TruSeq V3 chemistry kit (Illumina). Sequencing was performed on an Illumina HiSeq 2000 system. For each sample, we generated approximately 50 million paired-end reads 
at 101-bp length. Reads were trimmed for both adapters and low quality bases using Trimmomatic software and then aligned with the reference human hg19 genome and gene annotation from the Ensembl database (v70) using Tophat software. RNA mapping statistics were calculated using Picard software, and the average uniquely aligned reads were approximately $90 \%$ for each sample. We subsequently used the RNASeq workflow module in Partek Genomics Suite 6.6 (Partek Inc.; http:// www.partek.com/) and the R/Bioconductor package DESeq2 to identify differentially expressed genes at FDR less than $5 \%$ and a fold change $(\geq 1.5)$ cutoff. Partek performed an analysis based on RPKM units (reads per kilobase per million mapped reads) and the gene counts were fitted to a negative binomial generalized linear model with DESeq2. RNAseq data were deposited in the NCBI's Gene Expression Omnibus (GEO) database under accession number GSE80537.

ChIP coupled with high-throughput sequencing analysis of trimethylation at $\mathrm{H} 3 \mathrm{~K} 4 \mathrm{me}$. To examine genome-wide histone modification in ADHFE1- and MYC-overexpressing cells, we cultured MCF7 control cells and cells overexpressing these genes in triplicate and crosslinked DNA and protein directly in the culture medium with $1 \%$ formaldehyde solution for 10 minutes at room temperature. Chromatin was then fragmented to $150-200$ bp by 15 cycles of sonication (30 seconds on/30 seconds off) (Diagenode Bioruptor). Fragmented chromatin from $2 \times 10^{7}$ cells and $5 \mu \mathrm{g}$ of antibody against H3K4me3 (Abcam, ab8580) were used to perform the ChIP experiments using the High Sensitivity ChIP-IT Kit (Active Motif). Additional information can be found in the Supplemental Methods.

DNA methylation analysis using bisulfide sequencing. Bisulfide DNA libraries were constructed using the Accel-NGS Methyl-Seq DNA Library Kit from Swift Biosciences. Libraries were captured with The SeqCap Epi CpGiant Enrichment Kits from Roche NimbleGen, which covers more than 5.5 million $\mathrm{CpG}$ sites. Additional information can be found in the Supplemental Methods.

Pathway analysis. Gene lists from our analyses were derived from Partek and uploaded into the IPA tool to analyze for their relationship with IPA pathways by calculating pathway enrichment scores. IPA maintains a large-scale pathway network derived from the Ingenuity Knowledge Base, a large structured collection of observations in various experimental contexts with nearly 5 million findings manually curated from the biomedical literature or integrated from third-party databases. IPA calculates an enrichment score and $P$ values using the Fisher's exact test.

GSEA. GSEA was performed as previously described (55). This method identifies common features between gene expression data sets based on ranking metrics, measuring associations by a nonparametric, running-sum statistic, and estimates significance levels for the enrichment score. We applied GSEA to find common features between the gene signature associated with ADHFE1 amplification in TCGA breast tumors and signatures in the molecular signature database (http://www.broad.mit.edu/gsea/msigdb), such as the EMT signature reported by Sarrio et al. (26).

Analysis of MYC ChIP-seq data. Publicly available ChIP-seq data for MYC binding to genomic loci were queried in multiple cell lines using the ENCODE website (https://www.encodeproject.org/matrix /?type=Experiment\&status=released). The data for the ADHFE1 and AKR7A2 gene regions were visualized in the UCSC genome browser. Binding sites are shown as blue bars.

Analysis of the relationship between ADHFE1 protein expression and gene copy number alterations in breast tumors. TCGA breast cancer data for global mRNA and protein expression, and the gene copy number status in tumors, were obtained from cbioportal (http://www.cbioportal. org/). These data were previously published (14). Gene expression data were converted to $Z$ scores from RNAseq, protein data were converted to $Z$ scores from the mass spectrometry source, and copy number data were converted into copy number alterations using predicted copy number alterations from the GISTIC algorithm. Correlations between each feature were assessed using Spearman's rank correlation and are reported as the coefficient $\rho$ at a significance level of $P<0.05$.

Query of TCGA and METABRIC breast cancer data sets. We evaluated the publicly available TCGA and METABRIC breast cancer data accessible through the Cancer Genomics Data Server (CGDS; http:// www.cbioportal.org/public-portal) hosted by the Computational Biology Center at Memorial-Sloan-Kettering Cancer Center through C-Bioportal for Cancer Genomics. We queried somatic mutations, copy number alterations, and mRNA expression for a panel of genes of interest, including ADHFE1, MYC, IDH2, IDH1, and $P H G D H$, in the TCGA dataset. We also downloaded METABRIC data for ADHFE1 copy number alterations and patient survival. A survival analysis was performed comparing patients with and without an ADHFE1 amplification in their tumors, using Kaplan-Meier and Cox regression modeling in the R survival package.

Measurement of $2 \mathrm{HG}$ and 4-hydroxybutyrate. Total 2HG was measured as previously described (7). The relative abundance of $\mathrm{D}$ - and L-2HG in breast tumors and cultured cells was measured using chemical derivatization of $2 \mathrm{HG}$ as recently described (56). Additional information about these measurements and the quantification of 4-hydroxybutyrate in tumors can be found in the Supplemental Methods.

Metabolome analysis of cell lines. We analyzed the absolute concentration of 116 metabolites in cell lines using the metabolome analysis package Carcinoscope provided by Human Metabolome Technologies (HMT). For metabolite measurements by HMT, cell extracts were obtained following the manufacturer's protocol. Additional information can be found in the Supplemental Methods.

Metabolic flux of $\left[{ }^{13} \mathrm{C}\right]$ glutamine. The incorporation of $\left[{ }^{13} \mathrm{C}\right]$ glutamine into TCA cycle intermediates was determined as previously described (47). Additional information for the experiments with $\left[1{ }^{13} \mathrm{C}\right]$ glutamine can be found in the Supplemental Methods. To determine the flux of $\left[{ }^{13} \mathrm{C}\right]$ glutamine into succinate and citrate in presence and absence of cell-permeant 2HG, MCF12A cells were seeded in a 6-well plate and serum-starved overnight before being exposed to either $1 \mathrm{mM}$ octyl-2HG or control compound (PAMO) in RPMI medium containing $2 \mathrm{mM}\left[1-5{ }^{13} \mathrm{C}\right]$ glutamine (Cambridge Isotope Laboratories). Cells were washed with PBS and harvested after approximately 5 minutes, 12 hours, and 24 hours, counted, and were snap frozen using liquid nitrogen.

Western blot analysis. Total protein was extracted from the cells using RIPA lysis buffer with Halt protease inhibitor and protein concentration was estimated with the Bradford assay. Total protein (50 $\mu \mathrm{g}$ ) was resolved by SDS-PAGE in $10 \%$ gels followed by immunoblotting. Western blot analysis was performed using primary antibodies recognizing ADHFE1 (1:2,000; ADH8 S-16, Santa Cruz Biotechnology, sc-87546), MYC (1:250; 9E10, mouse monoclonal, Santa Cruz Biotechnology, sc-40), E-cadherin (1:5,000; BD Biosciences, 610182), N-cadherin (1:2,000; BD Biosciences, 610921), vimentin (1:2,000; Santa Cruz Biotechnology, sc-6260), ZEB1 (1:1,000; MilliporeSigma, HPA027524), SEMA4D (1:2,000; Abcam, ab134128), 
SEMA3E (1:2,000; Abcam, ab128619), PTK2/FAK (1:2,000; Cell Signaling Technology, 3285), ITGA4 (1:2,000; Abcam, ab81280), TFRC (1:1,000; Santa Cruz Biotechnology, sc32272), IRP2 (1:500; Santa Cruz Biotechnology, sc33682), ferritin (1:1,000; Abcam , ab183781), AKR1A1 (1:2,000; Abcam, ab125878); AKR7A2 (1:2,000; Abcam, ab97458), or actin (1:10,000; Calbiochem, CP01).

IHC. ADHFE1 protein expression by IHC was examined in 116 formalin-fixed and paraffin-embedded breast tumor tissues (50 ER-positive and 66 ER-negative tumors) from previously described patients (12) using a rabbit polyclonal anti-human ADHFE1 antibody (Santa Cruz Biotechnology, sc-87546) at 1:500 dilution. Antigen retrieval was performed using Dako Target Retrieval Solution pH 9.0 (Agilent Technologies). The primary antibody was applied at $4^{\circ} \mathrm{C}$ overnight in Dako background reducing diluent. After further incubation with anti-rabbit peroxidase-labeled polymer (30 minutes), ADHFE1 expression was visualized with the chromogen 3,3'-diaminobenzidine. The peroxidase blocking agent, peroxidase-labeled polymer, and the chromogen were part of the Dako EnVision System kit (Agilent Technologies). ADHFE1 expression in the tumor epithelium was scored as negative, low, moderate, or high using a standard scoring system as previously described $(12,57)$, and then categorized into low (negative to low) and high (moderate to high) for correlation and survival analysis. The IHC for E-cadherin was performed with the same general protocol but with the Dako mouse monoclonal anti-human E-cadherin antibody (clone NCH-38), 1:100 diluted. Expression of nuclear MYC was evaluated using a 1:200 dilution of the anti-human MYC antibody [Y69] from Abcam (ab32072).

Hypoxia treatment and D-2HG measurements. Cells $\left(1 \times 10^{6}\right)$ were seeded in $100-\mathrm{mm}$ petri dishes $(n=3)$ with regular culture media and incubated in a standard $\mathrm{CO}_{2}$ incubator with $21 \%$ oxygen, overnight. For hypoxia treatment, the cells were transferred into a hypoxia chamber supplied with $0.5 \%$ oxygen, $5 \%$ carbon dioxide, and $94.5 \%$ nitrogen, and maintained at $37^{\circ} \mathrm{C}$ and standard humidity. To measure intracellular and secreted D-2HG, cells were kept under hypoxia for 24 hours while the aerobically cultured cells were maintained in the standard incubator. Additional information can be found in the Supplemental Methods.

Mitochondrial ROS analysis. Cells were seeded in a 6-well plate at $2 \times 10^{5}$ cells per well with regular culture media. The next day, cells were treated for 48 hours with either $1 \mathrm{mM}$ octyl-2HG or control compound (PAMO) to determine if intracellular 2HG induces mitochondrial ROS. Alternatively, ADHFE1-overexpressing and vector control cells were grown for 72 hours and ROS was assessed. To measure mitochondrial ROS production, MitoSOX Red mitochondrial superoxide indicator (Molecular Probes, M36008) was applied to live cells. Cultured cells were washed with PBS and treated with $5 \mu \mathrm{M}$ MitoSOX Red for 30 minutes at $37^{\circ} \mathrm{C}$ without light exposure. As a positive control, cells were treated first with $10 \mu \mathrm{M}$ antimycin for 30 minutes before MitoSOX Red was added. Cells were then trypsinized and washed with PBS, and finally resuspended in PBS for the analysis by flow cytometry. Samples were run in the flow cytometer (FACSCanto II Bioanalyzer) at excitation $488 \mathrm{~nm}$ to measure oxidized MitoSOX Red and data analysis was performed using FlowJo version 8.5.2.

Iron measurements in mitochondria. Iron was measured by inductively coupled plasma-mass spectrometry (ICP-MS) using a NexION 350D (PerkinElmer) under DRC mode. Additional information can be found in the Supplemental Methods.
Migration and invasion assay. Migration and invasion were examined using the xCelligence System technology (Roche Applied Science/ACEA Biosciences, Inc.) for real-time monitoring of cellular processes with electronic cell sensor arrays, according to the manufacturer's instructions. Additional information can be found in the Supplemental Methods.

Analysis of EMT. To examine cellular morphology after treatment with cell-permeant D-2HG, $1 \times 10^{6}$ cells were plated in $60-\mathrm{mm}$ dishes, followed by treatment with either $1 \mathrm{mM}$ octyl-2HG or control compound (PAMO). Images of cells were taken at day 1 and 3 using a phase contrast microscope (Olympus IX51) at $\times 200$ magnification. These images were processed using the software ImageJ (version $1.50 \mathrm{i}$ at http://imagej.nih.gov/ij/). Similarly, to examine morphological changes induced by ADHFE1, $1 \times 10^{6}$ ADHFE1-overexpressing cells or vector control cells were plated in $60-\mathrm{mm}$ dishes and morphology was assessed at day 1 and 3. In experiments with $\mathrm{N}$-acetylcysteine, this antioxidant was added to cultured cells at a $10 \mathrm{mM}$ final concentration and images were taken after 24 hours of treatment. To examine morphological changes induced by ADHFE1 downregulation in MDA-MB-231 cells, cells were transfected with either negative control siRNA (Silencer Select Negative Control, 4390844, Thermo Fisher Scientific) or 2 siRNAs targeting ADHFE1 (Silencer Select siRNA-1 s44057, siRNA-2 s44059) following the manufacturer's protocol using Lipofectamine RNAiMAX. Changes in morphology were assessed 3 days after the transfections. For additional treatment with cell-permeant D-2HG, MDA-MB-231 cells were first transfected with siRNA, cultured for 1 day, and then exposed to $1 \mathrm{mM}$ octyl-2HG for an additional 2 days before morphology was assessed. A cell that showed loss of cell-cell adhesion and a spindle-shaped morphology was scored as a mesenchymal cell in the experiments with the MCF7, MCF10A, and MCF12A cells. When scoring the morphology of MDA-MB-231 cells, spindle-shaped or stellate cells with elongated protrusions were scored as mesenchymal-like, whereas round cells or cells with a cobblestone appearance were scored as epithelial-like. Experiments were performed in triplicate and percentage of altered morphology among the total cell population was calculated (per 100 evaluated cells), using cell counts from representative fields across experiments and scoring at least 500 cells per experimental group.

Sphere formation assay. MCF10A and MCF12A cells with or without $A D H F E 1$ overexpression were seeded (1,000 cells per 100 $\mu \mathrm{l}$ of Matrigel/media, 50:50) at the center of a microwell in 35-mm glass-bottom culture plates (MatTek), followed by a 1-hour incubation in $37^{\circ} \mathrm{C}$ and addition of $2 \mathrm{ml}$ of regular media to the plates. For experiments with $2 \mathrm{HG}$ treatment, the medium was supplemented with either $1 \mathrm{mM}$ octyl-2HG or control compound (PAMO). The formation of spheres and their growth were monitored daily. After 2 weeks, the spheres were counted under a phase contrast microscope at $\times 40$ magnification and quantified using ImageJ.

Statistics. All statistical tests were 2-sided and an association was considered statistically significant at $P$ less than 0.05 . Statistical analyses were performed either in Prism 6 (GraphPad) or with the $\mathrm{R}$ software developed by R Development Core Team at R Foundation for Statistical Computing and packages in Bioconductor (https:// www.r-project.org). We generated Kaplan-Meier survival plots and used the log-rank test for significance testing. The Cox proportional hazards model was applied to estimate unadjusted and adjusted hazard ratios and 95\% confidence intervals for breast cancer survival. 
Study approval. The collection of biospecimens and the clinical and pathological information was approved by the University of Maryland IRB for the participating institutions (UMD protocol 0298229). IRB approval of this protocol was then obtained at all institutions (Veterans Affairs Medical Center, Union Memorial Hospital, Mercy Medical Center, and Sinai Hospital, Baltimore, Maryland, USA). The research was also reviewed and approved by the NIH Office of Human Subjects Research Protections (OHSRP 2248). All patients signed a consent form. The described animal procedures were reviewed and approved by the NCI-Bethesda Animal Care and Use Committee (IACUC protocol number LHC-004-2). NCI is accredited by AAALAC International and follows the Public Health Service Policy for the Care and Use of Laboratory Animals.

\section{Author contributions}

PM, AS, NP, and SA conceived and designed the study. PM, WT, VP, THD, FJ, FW, DZ, LA, TD, SZ, JKK, and TZM acquired the data. PM, WT, LA, YW, HGY, WL, and NP analyzed the data. DHL,
AS, MB, WL, NP, and SA provided resources. AS, MB, WL, NP, and SA supervised the study. PM, VP, WT, THD, MS, AS, WL, NP, and SA wrote and reviewed the manuscript and/or revised data.

\section{Acknowledgments}

This research was supported by the Intramural Research Program of the NIH, NCI, Center for Cancer Research. In addition, grants to Arun Sreekumar and Nagireddy Putluri supported this work (W81XWH-12-546 1-0130 from the Department of Defense; DMS 1161759 from the National Science Foundation; NIH U01 CA167234, R21-CA185516-01; NCI 2P30CA125123-09, 127430-RSG-15-105-01-CNE; and CPRIT Metabolomics Core Facility Support Award RP120092 Alkek CMD grants).

Address correspondence to: Stefan Ambs, National Cancer Institute, Building 37/Room 3050B, Bethesda, Maryland 20892-4258, USA. Phone: 240.760.6836; Email: ambss@mail.nih.gov.
1. Locasale JW, et al. Phosphoglycerate dehydrogenase diverts glycolytic flux and contributes to oncogenesis. Nat Genet. 2011;43(9):869-874.

2. Possemato R, et al. Functional genomics reveal that the serine synthesis pathway is essential in breast cancer. Nature. 2011;476(7360):346-350.

3. Sullivan LB, Gui DY, Heiden MGV. Altered metabolite levels in cancer: implications for tumour biology and cancer therapy. Nat Rev Cancer. 2016;16(11):680-693.

4. DeBerardinis RJ, Chandel NS. Fundamentals of cancer metabolism. Sci Adv. 2016;2(5):e1600200.

5. Mishra P, Ambs S. Metabolic signatures of human breast cancer. Mol Cell Oncol. 2015;2(3):e992217.

6. Budczies J, et al. Comparative metabolomics of estrogen receptor positive and estrogen receptor negative breast cancer: alterations in glutamine and beta-alanine metabolism. J Proteomics. 2013;94:279-288

7. Terunuma A, et al. MYC-driven accumulation of 2-hydroxyglutarate is associated with breast cancer prognosis. J Clin Invest. 2014;124(1):398-412.

8. Tang X, Lin CC, Spasojevic I, Iversen ES, Chi JT, Marks JR. A joint analysis of metabolomics and genetics of breast cancer. Breast Cancer Res. 2014;16(4):415.

9. Dang L, et al. Cancer-associated IDH1 mutations produce 2-hydroxyglutarate. Nature. 2009;462(7274):739-744.

10. Ward PS, et al. The common feature of leukemiaassociated IDH1 and IDH2 mutations is a neomorphic enzyme activity converting alphaketoglutarate to 2-hydroxyglutarate. Cancer Cell. 2010;17(3):225-234.

11. Pereira B, et al. The somatic mutation profiles of 2,433 breast cancers refines their genomic and transcriptomic landscapes. Nat Commun. 2016;7:11479.

12. Boersma BJ, et al. Association of breast cancer outcome with status of p53 and MDM2 SNP309. J Natl Cancer Inst. 2006;98(13):911-919.

13. Kessler JD, et al. A SUMOylation-dependent transcriptional subprogram is required for Myc-driven tumorigenesis. Science. 2012;335(6066):348-353.

14. Mertins P, et al. Proteogenomics connects somatic mutations to signalling in breast cancer.
Nature. 2016;534(7605):55-62.

15. Wu KJ, Polack A, Dalla-Favera R. Coordinated regulation of iron-controlling genes, $\mathrm{H}$-ferritin and IRP2, by c-MYC. Science. 1999;283(5402):676-679.

16. Tang H, et al. Downregulation of HSP60 disrupts mitochondrial proteostasis to promote tumorigenesis and progression in clear cell renal cell carcinoma. Oncotarget. 2016;7(25):38822-38834

17. Lyon RC, Johnston SM, Panopoulos A, Alzeer S, McGarvie G, Ellis EM. Enzymes involved in the metabolism of gamma-hydroxybutyrate in SH-SY5Y cells: identification of an irondependent alcohol dehydrogenase ADHFe1. Chem Biol Interact. 2009;178(1-3):283-287.

18. Struys EA, Verhoeven NM, Ten Brink HJ, Wickenhagen WV, Gibson KM, Jakobs C. Kinetic characterization of human hydroxyacid-oxoacid transhydrogenase: relevance to D-2-hydroxyglutaric and gamma-hydroxybutyric acidurias. JInherit Metab Dis. 2005;28(6):921-930.

19. Watson E, et al. Metabolic network rewiring of propionate flux compensates vitamin B12 deficiency in C. elegans. Elife. 2016;5:e17670.

20. Lyon RC, Johnston SM, Watson DG, McGarvie G, Ellis EM. Synthesis and catabolism of gammahydroxybutyrate in SH-SY5Y human neuroblastoma cells: role of the aldo-keto reductase AKR7A2. J Biol Chem. 2007;282(36):25986-25992.

21. Wise DR, et al. Hypoxia promotes isocitrate dehydrogenase-dependent carboxylation of $\alpha$-ketoglutarate to citrate to support cell growth and viability. Proc Natl Acad Sci U S A 2011;108(49):19611-19616.

22. Metallo CM, et al. Reductive glutamine metabolism by IDH1 mediates lipogenesis under hypoxia. Nature. 2011;481(7381):380-384.

23. Oster B, et al. Identification and validation of highly frequent $\mathrm{CpG}$ island hypermethylation in colorectal adenomas and carcinomas. Int $J$ Cancer. 2011;129(12):2855-2866.

24. Kruger RP, Aurandt J, Guan KL. Semaphorins command cells to move. Nat Rev Mol Cell Biol. 2005;6(10):789-800.

25. Zhou Y, Gunput RA, Pasterkamp RJ. Semaphorin signaling: progress made and promises ahead.
Trends Biochem Sci. 2008;33(4):161-170.

26. Sarrió D, Rodriguez-Pinilla SM, Hardisson D, Cano A, Moreno-Bueno G, Palacios J. Epithelial-mesenchymal transition in breast cancer relates to the basal-like phenotype. Cancer Res. 2008;68(4):989-997.

27. Radisky DC, et al. Rac1b and reactive oxygen species mediate MMP-3-induced EMT and genomic instability. Nature. 2005;436(7047):123-127.

28. Xu W, et al. Oncometabolite 2-hydroxyglutarate is a competitive inhibitor of $\alpha$-ketoglutaratedependent dioxygenases. Cancer Cell. 2011;19(1):17-30.

29. Chowdhury R, et al. The oncometabolite 2-hydroxyglutarate inhibits histone lysine demethylases. EMBO Rep. 2011;12(5):463-469.

30. Colvin H, et al. Oncometabolite D-2-hydroxyglurate directly induces epithelial-mesenchymal transition and is associated with distant metastasis in colorectal cancer. Sci Rep. 2016;6:36289.

31. Berger SL. The complex language of chromatin regulation during transcription. Nature. 2007;447(7143):407-412.

32. Tenhagen $M$, van Diest PJ, Ivanova IA, van der Wall E, van der Groep P. Fibroblast growth factor receptors in breast cancer: expression, downstream effects, and possible drug targets. Endocr Relat Cancer. 2012;19(4):R115-R129.

33. Guo W, et al. Slug and Sox 9 cooperatively determine the mammary stem cell state. Cell. 2012;148(5):1015-1028.

34. Wang H, et al. SOX9 regulates low density lipoprotein receptor-related protein 6 (LRP6) and T-cell factor 4 (TCF4) expression and Wnt/ $\beta$-catenin activation in breast cancer. J Biol Chem. 2013;288(9):6478-6487.

35. Jeselsohn R, et al. Embryonic transcription factor SOX 9 drives breast cancer endocrine resistance. Proc Natl Acad Sci U S A. 2017;114(22):E4482-E4491.

36. Figueroa ME, et al. Leukemic IDH1 and IDH2 mutations result in a hypermethylation phenotype, disrupt TET2 function, and impair hematopoietic differentiation. Cancer Cell. 2010;18(6):553-567.

37. Grassian AR, et al. Isocitrate dehydrogenase 
(IDH) mutations promote a reversible ZEB1/ microRNA (miR)-200-dependent epithelialmesenchymal transition (EMT). J Biol Chem. 2012;287(50):42180-42194.

38. $\mathrm{Lu} \mathrm{C}$, et al. IDH mutation impairs histone demethylation and results in a block to cell differentiation. Nature. 2012;483(7390):474-478.

39. Fathi AT, et al. Isocitrate dehydrogenase 1 (IDH1) mutation in breast adenocarcinoma is associated with elevated levels of serum and urine 2-hydroxyglutarate. Oncologist. 2014;19(6):602-607.

40. Shim EH, et al. L-2-Hydroxyglutarate: an epigenetic modifier and putative oncometabolite in renal cancer. Cancer Discov. 2014;4(11):1290-1298.

41. Intlekofer AM, et al. Hypoxia induces production of L-2-hydroxyglutarate. Cell Metab. 2015;22(2):304-311.

42. Oldham WM, Clish CB, Yang Y, Loscalzo J. Hypoxia-mediated increases in L-2-hydroxyglutarate coordinate the metabolic response to reductive stress. Cell Metab. 2015;22(2):291-303.

43. McCleland ML, et al. An integrated genomic screen identifies LDHB as an essential gene for triple-negative breast cancer. Cancer Res. 2012;72(22):5812-5823.

44. Fan J, et al. Human phosphoglycerate dehydroge- nase produces the oncometabolite D-2-hydroxyglutarate. ACS Chem Biol. 2015;10(2):510-516.

45. Smolková K, Dvořák A, Zelenka J, Vítek L, Ježek P. Reductive carboxylation and 2-hydroxyglutarate formation by wild-type IDH2 in breast carcinoma cells. Int J Biochem Cell Biol. 2015;65:125-133.

46. Fendt SM, et al. Reductive glutamine metabolism is a function of the $\alpha$-ketoglutarate to citrate ratio in cells. Nat Commun. 2013;4:2236.

47. Dasgupta S, et al. Coactivator SRC-2-dependent metabolic reprogramming mediates prostate cancer survival and metastasis. J Clin Invest. 2015;125(3):1174-1188.

48. Mullen AR, et al. Oxidation of alpha-ketoglutarate is required for reductive carboxylation in cancer cells with mitochondrial defects. Cell Rep. 2014;7(5):1679-1690.

49. Leonardi R, Subramanian C, Jackowski S, Rock CO. Cancer-associated isocitrate dehydrogenase mutations inactivate NADPHdependent reductive carboxylation. J Biol Chem. 2012;287(18):14615-14620.

50. Grassian AR, et al. IDH1 mutations alter citric acid cycle metabolism and increase dependence on oxidative mitochondrial metabolism. Cancer Res. 2014;74(12):3317-3331.
51. Sciacovelli M, et al. Fumarate is an epigenetic modifier that elicits epithelial-to-mesenchymal transition. Nature. 2016;537(7621):544-547.

52. Losman JA, et al. (R)-2-hydroxyglutarate is sufficient to promote leukemogenesis and its effects are reversible. Science. 2013;339(6127):1621-1625.

53. Kim JY, Tillison KS, Zhou S, Lee JH, Smas CM. Differentiation-dependent expression of Adhfe1 in adipogenesis. Arch Biochem Biophys. 2007;464(1):100-111.

54. Marques O, da Silva BM, Porto G, Lopes C. Iron homeostasis in breast cancer. Cancer Lett. 2014;347(1):1-14.

55. Subramanian A, et al. Gene set enrichment analysis: a knowledge-based approach for interpreting genome-wide expression profiles. Proc Natl Acad Sci U S A. 2005;102(43):15545-15550.

56. Struys EA, Jansen EE, Verhoeven NM, Jakobs C. Measurement of urinary D- and L-2-hydroxyglutarate enantiomers by stable-isotope-dilution liquid chromatography-tandem mass spectrometry after derivatization with diacetyl-L-tartaric anhydride. Clin Chem. 2004;50(8):1391-1395.

57. Prueitt RL, et al. Inflammation and IGF-I activate the Akt pathway in breast cancer. Int J Cancer. 2007;120(4):796-805. 